\title{
Occurrence and predictive risk factors associated with in-stent restenosis after drug-eluting stent implantation in diabetic patients: a prospective, clinical cohort study
}

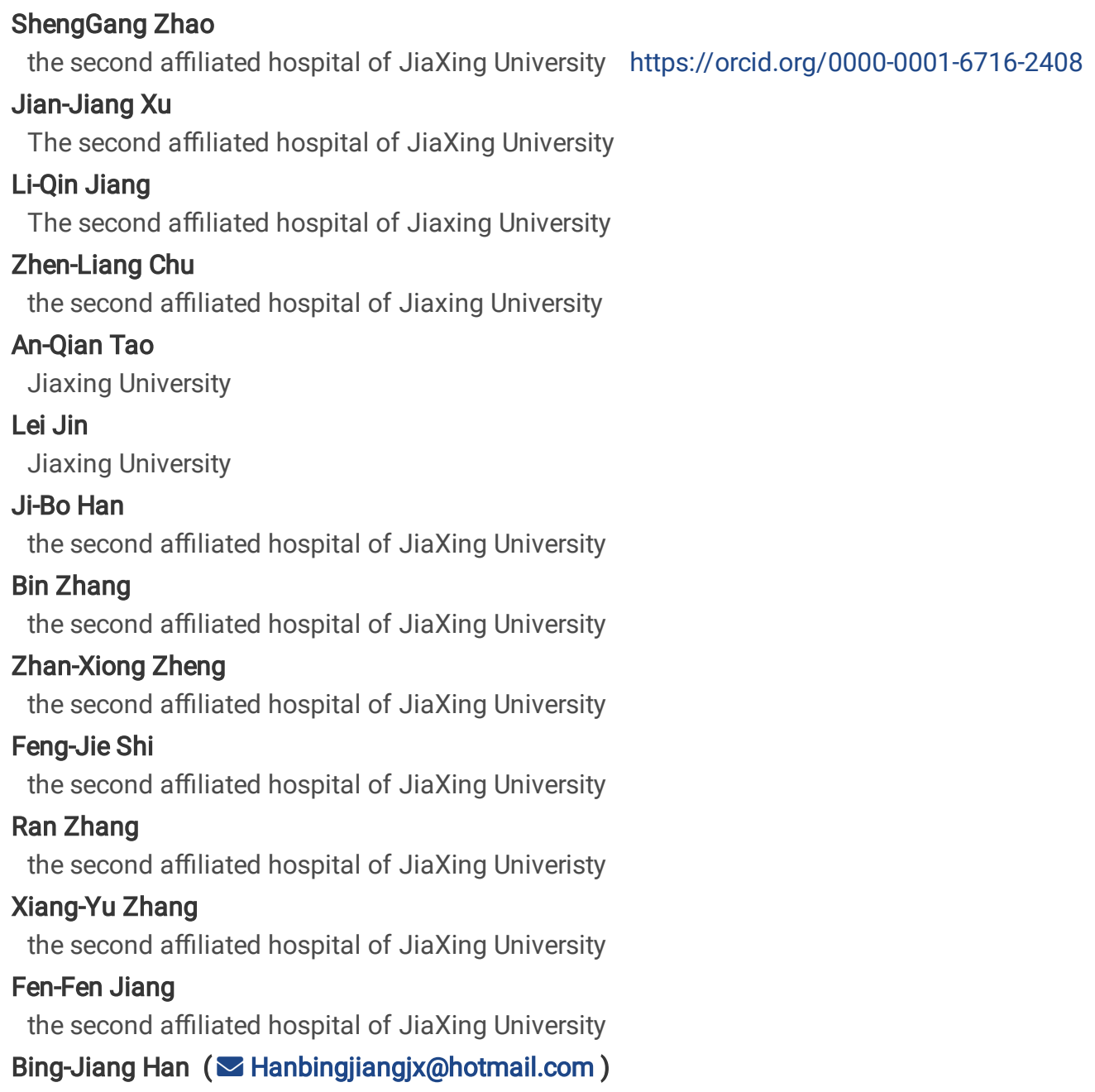

Original investigation

Keywords: Diabetes mellitus, In-stent restenosis, Lipids, Inflammation

Posted Date: May 15th, 2020

DOI: https://doi.org/10.21203/rs.3.rs-26601/v1

License: (c) This work is licensed under a Creative Commons Attribution 4.0 International License. Read Full License 


\section{Abstract \\ Background}

The incidence of in-stent restenosis (ISR) in patients with diabetes mellitus (DM) after percutaneous coronary intervention (PCI) is significantly higher than that in patients without DM, but the mechanism is not clear. We hypothesised that patients with and risk factors including dyslipidaemia, elevated inflammatory factors would be prone to induction of ISR, and that dynamic observation of the comprehensive risk factor changes before and after PCl would be helpful to identify ISR .

\section{Methods}

This prospective cohort study consecutively enrolled 360 patients who received coronary drug-eluting stent implantation. Patients who underwent coronary angiography (CAG) and received clinical follow-up were prospectively reviewed. The patients were assigned to a DM (262) or a non-DM (98) group. The patients were further assigned according to whether ISR was present to the non-DM + non-ISR, non-DM + ISR, DM + non-ISR, and DM + ISR groups. The patients were further assigned according to whether low-density lipoprotein (LDL-c) was decreased more than $50 \%$ compared with baseline, or was less than $1.80 \mathrm{mmol} / \mathrm{L}$ in the follow-up, to the LDL-c achieved or the LDL-c failure groups.

\section{Results}

DM patients were prone to develop ISR after $\mathrm{PCl}$ and the degree of coronary stenosis was more severe than in non-DM patients. This result was more striking in DM and LDL-c failure patients. The levels of total cholesterol (TC), triglyceride, high-density lipoprotein (HDL-C), LDL-C, apolipoprotein B100, apolipoprotein E, remnant lipoprotein, TC/HDL-c ratio and triglyceride/HDL-c ratio in the DM + non-ISR were similar to those in the DM + ISR group before PCl and CAG. The DM + ISR group had the highest levels of haemoglobin A1c and the highest Gensini scores. The inflammatory index changes including leukocytes and neutrophils were the most striking in the DM + ISR group. In multivariate regression analysis, neutrophil changes and glycosylated haemoglobin were independent risk factors for ISR [ $\triangle$ neutrophil, OR $1.929,95 \%$ Cl 1.216-3.058; HbA1-c OR 1.559,95\% Cl 1.001-1.707].

\section{Conclusion}

Coronary artery disease patients with DM had a high risk for ISR if they had preoperative risk factors including dyslipidaemia, elevated inflammatory factors, and a high Gensini score. Dynamic observation of the changes of the preoperative and postoperative comprehensive risk factors was helpful to identify ISR in patients with DM.

\section{Background}

Percutaneous coronary intervention ( $\mathrm{PCl}$ ) is the most commonly used clinical method for the treatment of coronary atherosclerosis [1]. However, in-stent restenosis (ISR) has become a thorny problem in clinical practice. Although several large clinical trials showed that drugeluting stents (DES) could significantly reduce the rate of ISR [2], the incidence of ISR after DES has remained at $5 \%-10 \%$. Despite the adoption of new drugs and revascularisation strategies, ISR after PCl remains a major obstacle for interventional cardiology

ISR is defined as a gradual re-narrowing of a stented lumen due to arterial damage and subsequent tissue proliferation. The ISR definition is further divided into coronary angiography (CAG) ISR and clinical ISR [3]. The ISR defined by CAG is a lumen diameter that is reduced by $50 \%$ or more in the stent, or within $5 \mathrm{~mm}$ of the periphery of the stent, as determined by follow-up angiography after PCI [2, 3]. Clinical ISR is defined based on the presence of a $50 \%$ or more reduction in the coronary artery lumen diameter within $5 \mathrm{~mm}$ of the stent implantation or the periphery of the stent, and when one or more of the following conditions is also present: evidence of objective ischaemia (electrocardiogram changes); history of recurrent angina; coronary fractional flow reserve value $<0.8$; intravascular ultrasonography detected minimum cross-sectional vascular area $<4 \mathrm{~mm}^{2}$ (Left trunk $<6 \mathrm{~mm}^{2}$ ); and restenosis that exceeds more than $70 \%$ reduction in the diameter of the lumen even without obvious symptoms [3].

As coronary stents have evolved from bare metal stents (BMS) to DES, then to subsequent DES iterations, the incidence of ISR has gradually decreased. J-Cypher research focused on the adverse reactions of the first generation DES and enrolled 12,812 patients who received sirolimus-eluting stent treatment [3]. Among these patients, the 1-year target lesion revascularisation rate was $7.3 \%$, at 5 years it 
was $15.9 \%$, and the annual incidence rate was $2.2 \%$ [4]. Compared with the first generation DES, the incidence of all-cause death and myocardial infarction in the second-generation DES was significantly reduced [5]. However, in the first and second generations of DES, there was a late catch-up phenomenon of endometrial hyperplasia, which resulted in a significant increase in the incidence of late occurring ISR [1]. The reason why the incidence of ISR has increased significantly in recent years may be that patients with complicated and serious coronary artery disease received PCI treatments, and had high Syntax scores, which caused a significant increase in the restenosis rate $[1,2,6]$.

Patients with DM have a significantly higher percentage of ISR after PCI than that of patients without DM. The mechanism may be that diabetes can lead to an abnormal function of the vascular endothelium and smooth muscle cells, which can promote thrombosis and platelet aggregation [7, 8], hyperglycaemia, excessive free fatty acid release, insulin resistance, and an exacerbation of vascular endothelial dysfunction. These factors can lead to increased activity of proinflammatory transcription factors, overexpression of leukocyte adhesion molecules, and the production of chemokines and cytokines. Hyperglycaemia can enhance the activity of advanced glycation end products, thereby accelerating vascular inflammation, promoting smooth muscle cell proliferation and extracellular matrix production, and ultimately lead to excessive intimal hyperplasia and restenosis [8]. In addition, due to diabetic atherosclerosis, which is often complicated by insufficient vascular compensatory remodelling, patients with DM often have blood vessels with small diameters [9]. Insufficient stent expansion is also a mechanism for the formation of diabetic ISR $[10,11]$. In addition, dyslipidaemia is common in patients with DM, and is characterised by high triglyceride (TG) and VLDL-c levels, along with normal LDL-c levels [12].

At present, there are many studies that have examined ISR and baseline blood lipid composition, but there are few studies that have focused on the predictive value of blood lipid composition changes and the development of ISR. In addition, dyslipidaemia is closely related to the inflammatory response, and is clearly correlated with ISR. Because patients with DM often have dyslipidaemia and hyperinflammation, further exploration of the comprehensive risk factors regarding the occurrence of ISR in DM, and the identification of possible targets and prediction models is necessary. Several studies have focused on the level of risk factors at baseline before $\mathrm{PCl}$ to predict ISR, and few studies examined the changes in the levels of lipids, glucose, and inflammation to explore ISR in patients with DM. Furthermore, most previous studies were retrospective. Additionally, the interaction relationship of many risk factors for stent restenosis was not clear. We therefore hypothesised that 1 ) patients with coronary artery disease who had risk factors including dyslipidaemia, elevated inflammatory factors, and high Gensini scores would be prone to develop ISR, and 2) that dynamic observation of the changes of comprehensive risk factors before and after PCl would be helpful for the early identification of ISR in patients with DM.

\section{Methods}

\section{Study population}

This prospective clinical cohort study continuously enrolled stable angina or acute coronary syndrome patients who underwent DES implantation, and who were expected to undergo CAG repeated in clinical follow-up in the Department of Cardiology at the the second affiliated hospital of Jiaxing University from January 2012 to December 2017. The aim of the study was to determine whether patients with coronary artery disease who had risk factors including dyslipidaemia, elevated inflammatory factors, and high Gensini scores would be more likely to develop ISR, and to determine if the dynamic observation of the changes of comprehensive risk factors before and after $\mathrm{PCl}$ would be helpful for the early identification of ISR in patients with DM.

The inclusion criteria were: (1) patients older than 18 years; (2) patients who were diagnosed in accordance with the diagnostic criteria for coronary intervention therapy [13]; (3) patients with an initial stent treatment; (4) patients with a successful stent implantation, and residual stenosis that was less than $20 \%$; (5) patients who experienced no serious complications during the operation and hospitalisation; and (6) long-term (at least one year) tolerance of dual antiplatelet drugs, and statin therapy after discharge.

The exclusion criteria were: (1) patients with a previous history of coronary stent treatment and coronary artery bypass grafting; (2) patients with a previous restenosis after coronary drug balloon therapy; (3) patients with a history of malignant tumour, abnormal liver and kidney function, or with infectious diseases or acute and chronic active inflammation; (4) patients who were allergic to aspirin, clopidogrel, statins, heparin and other drugs; and (5) patients who had a history of haemolytic diseases, rheumatism, and thyroid hormone abnormalities.

Because this was a prospective study, all subjects were required to sign an informed consent form. The study complied with the Declaration of Helsinki and was approved by the ethics committee of the second affiliated hospital of Jiaxing University.

\section{Research plan}




\section{Grouping}

According to the exposure factors for DM, all patients were assigned to the combined DM group or to the non-combined DM group. The patients' baseline parameters included age, sex, the presence of risk factors related to CHD (DM, hypertension, dyslipidaemia, family history, and smoking), fasting blood glucose, thyroid function, blood electrolytes, blood lipid profile, left ventricular ejection score (LVEF), and cardiac ultrasound results were collected. Hypertension was defined as a history of systolic blood pressure (SBP) $>140 \mathrm{mmHg}$ and / or diastolic blood pressure (DBP) $>90 \mathrm{mmHg}$. DM was defined by fasting blood glucose test results of at least $126 \mathrm{mg} / \mathrm{dl}$ and / or the use of insulin or other antidiabetic drugs.

Fasting venous blood was tested before PCl and before repeated CAG in follow-up. The specific indicators included: TC, TG, LDL-C, HDL-C, RPL-C (TC minus low-density lipoprotein and high-density lipoprotein), non-HDL-c (TC minus high-density lipoprotein), ApoA1, ApoB100, ApoE, uric acid (UC), creatinine (Ccr), blood calcium concentration, blood magnesium concentration, blood phosphorus concentration, homocysteine ( Hcy), Glycated haemoglobin A1c (HbA1c), C-reactive protein (CRP), white blood cell (WBC), neutrophil (N), lymphocyte (L), monocyte $(M)$, red blood cell $(R B C)$, haemoglobin $(\mathrm{Hb})$, platelet value (PLT), RBC distribution width (RDW), and platelet distribution width (PDW).

\section{Interventional treatment of coronary heart disease}

Patients who underwent elective interventional therapy were administered aspirin $0.1 \mathrm{~g}$, clopidogrel $75 \mathrm{mg}$, and atorvastatin $20 \mathrm{mg}$ once a day 48 hours before surgery. Emergency intervention patients were administered aspirin $0.3 \mathrm{~g}$, clopidogrel $600 \mathrm{mg}$ and atorvastatin $40 \mathrm{mg}$ once as a loading dose. We followed the standardised procedures for interventional treatment guidelines for stable CHD and unstable coronary heart disease ${ }^{[13]}$. The criteria for successful stent implantation included: complete stent expansion, residual stenosis $<20 \%$; no obvious damage to the proximal and distal vessels of the stent; and thrombolysis in myocardial infarction (TIMI) blood flow level 3. The PCI-related DES stent diameter, the length and balloon inflation pressure, and the postoperative standardised medical treatment were recorded.

The Gensini score was calculated based on the severity of coronary stenosis as shown by CAG as follows: $1 \%$ to $25 \%$ coronary stenosis was scored as one point, $26 \%$ to $50 \%$ stenosis was two points, $51 \%$ to $75 \%$ stenosis was four points, $76 \%$ to $90 \%$ was eight points, $91 \%$ to $99 \%$ was 19 points, and 100\% complete occlusion was 32 points. The coefficients of the corresponding lesions and branch vessels were assessed as follows: 5 points for the left main trunk, 2.5 points for the anterior descending branch, 1.5 points for the middle segment, 1.5 points for the distal segment, 1 point for the first diagonal branch, 1 point for the second diagonal branch, 2.5 points for the proximal segment of the circumflex branch, 1 point for the distal segment, 1 point for the blunt margin branch, and 1 point each for the proximal segment of the right coronary artery, middle segment, distal segment, posterior descending branch, and posterior branch of the left ventricle. All the lesions were identified, then they were multiplied by the corresponding coefficient; the integrated score was the total Gensini score. The Gensini score (no-stent) was the total Gensini score minus the coronary with stent implantation Gensini score. The participants were assigned to the LDL-c achieved group or the LDL-c failure group according to whether LDL-c was decreased by more than $50 \%$ compared with baseline or was less than $1.80 \mathrm{mmol} / \mathrm{L}$ in the follow-up.

\section{Clinical follow-up and CAG}

After being discharged from the hospital, the patients were followed up by telephone or an outpatient clinic visit once a month to record the health status, medication use, and to inquire about the side effects and symptoms the patients have experienced. The end point was set as restenosis in the stent.

The CAG results were routinely reviewed 1 year after PCI in the enrolled population. If patients experienced clinical symptoms (typical angina symptoms, positive exercise plate test, acute coronary syndrome, among others), they could elect to undergo a repeat CAG. Two independent interventional physicians interpreted the CAG results. If the results were inconsistent, three or more interventional physicians provided a joint interpretation. CAG was performed with a digital subtraction angiography system (Allura Xper FD-20 X-ray system; Philips Medical, Netherlands). CAG ISR was determined as follows: re-angiography revealed that the stent or the surrounding segments of the stent (within $5 \mathrm{~mm}$ of the two sides of the stent) lumen diameter was narrowed by more than $50 \%$. If $<50 \%$ stenosis was observed, it was interpreted as non-ISR.

\section{Statistical analysis}

We used SPSS statistical software package, version 22.0 (IBM, New York, United States) to conduct the statistical analyses. The data distribution was analysed by a Kolmogorov-Smirnov test. When a normal distribution was met, the continuous variable data was expressed 
as a mean and standard deviation, and the non-normal data was expressed as a median (interquartile range). Continuous variables were tested using the independent Student test, Mann-Whitney U test or Wilcoxon rank sum test. The categorical variables were expressed as rates (\%), and the chi-square test was used. Univariate and multivariate regression analysis was used to assess the association between the risk factors and ISR. The Receiver operating characteristic curve, (ROC) was used to determine the optimal cut-off value of the risk factors that could predict ISR. The Kaplan-Meier method was used to analyse the cumulative occurrence of ISR in the DM and non-DM groups, along with a log-rank calculation test. A two-sided $P$ value $<0.05$ was determined to be statistically significant.

\section{Results}

\section{Follow-up PCl population baseline and interventional treatment comparison}

A total of 400 follow-up PCI patients were enrolled [non-diabetic group ( $n=284)$,

diabetic group ( $n=116)$ ], 40 patients missing from follow-up, included 22 in the non-diabetic and 18 in the diabetic group(Supplementary additional file Fig. 1). Then total of 360 patients with follow-ups included 262 in the non-DM group and 98 in the DM group. Table 1 shows the disease composition in the non-DM and the DM groups (stable angina, acute coronary syndrome proportion), and left ventricular ejection fraction, left atrium diameter, stent length, average balloon dilation pressure, follow-up interval. There is no statistical difference in the data shown in Table 1. However, the DM group included more participants with hypertension compared to the non-DM group ( $\mathrm{P}<0.05)$. There were more patients in the DM group who did not achieve the systolic blood pressure control target compared to the non-DM group ( $\mathrm{P}$ $<0.05)$. The DM group included fewer smokers $(41 / 98)$ compared to the non-DM group $(145 / 262)(P<0.05)$. 
Table 1

Comparison of baseline and interventional treatment in $\mathrm{PCl}$ population

\begin{tabular}{|c|c|c|c|}
\hline & Non-DM(n = 262) & $\mathrm{DM}(\mathrm{n}=98)$ & $\mathbf{P}$ \\
\hline Age (years) & $63.89 \pm 9.93$ & $65.19 \pm 10.63$ & 0.268 \\
\hline Male, n (\%) & 196((74.81\%) & $62(63.27 \%)$ & 0.031 \\
\hline Hypertension, n (\%) & 186((70.99\%) & $79(80.61 \%)$ & 0.031 \\
\hline Systolic blood pressure $\mathrm{mmHg}$ & $135.65 \pm 21.57$ & $141.22 \pm 20.19$ & 0.027 \\
\hline Diastolic blood pressure, $\mathrm{mmHg}$ & $78.88 \pm 12.87$ & $80.38 \pm 12.76$ & 0.326 \\
\hline Smoking history, n (\%) & $145(55.34 \%)$ & $41(41.84 \%)$ & 0.022 \\
\hline Hyperlipidemia, n (\%) & $72(27.01 \%)$ & $30(30.61 \%)$ & 0.571 \\
\hline SAP & 119 & 44 & 0.992 \\
\hline ACS & 143 & 54 & \\
\hline LVEF(\%) & $61.50 \pm 6.84$ & $61.16 \pm 7.63$ & 0.718 \\
\hline $\mathrm{LA}(\mathrm{mm})$ & $34.48 \pm 5.10$ & $35.07 \pm 4.61$ & 0.376 \\
\hline \multicolumn{4}{|l|}{ Stent target vessel position, $\mathrm{n}(\%)$} \\
\hline Left anterior descending artery, n (\%) & $155(59.16 \%)$ & $63(64.29 \%)$ & \multirow[t]{4}{*}{0.039} \\
\hline Right coronary artery, n (\%) & $60(22.90 \%)$ & $29(29.59 \%)$ & \\
\hline Left circumflex artery, n (\%) & $38(14.50 \%)$ & $5(5.10 \%)$ & \\
\hline Left main trunk, n (\%) & $9(3.44 \%)$ & $1(1.02 \%)$ & \\
\hline Stent diameter (mm) & $3.00(1.50 \rrbracket 4.00)$ & $3.00(2.25 \rrbracket 4.00)$ & 0.127 \\
\hline Stent length (mm) & $24.00(12.00 \otimes 38.00)$ & $12.00(12.00 \otimes 38.00)$ & 0.485 \\
\hline Average balloon inflation pressure, atm & $15.00(8.00 \bowtie 26.00)$ & $16.00(9.00 \rrbracket 22.00)$ & 0.148 \\
\hline Follow-up interval (months) & $12.00(1.00-77.00)$ & $12.00(1.00-72.00)$ & 0.520 \\
\hline \multicolumn{4}{|c|}{ Data were expressed as mean \pm SD, median with minimum and maximum or $\mathrm{n}(\%)$} \\
\hline
\end{tabular}

\section{Comparison of the incidence of intra-stent restenosis at the end of follow-up}

ISR accounted for $20 / 98$ vs $27 / 262$ in the DM and non-DM populations, respectively $(\mathrm{P}<0.05)$ (Supplementary additional file Fig. 2 ). Therefore, according to the history of DM and whether ISR occurred, participants were assigned to the non-DM + non-ISR group ( $\mathrm{n}=235)$, non-diabetes + ISR group $(\mathrm{n}=27)$, DM + Non-ISR group $(\mathrm{n}=78)$, and DM + ISR group $(\mathrm{n}=20)$.

The patients' baseline characteristics and disease composition were similar in the four groups. However, in the two groups with DM (DM + non-ISR, and DM + ISR), the proportion of patients with hypertension was higher than that of the non-DM groups (non-DM + non-ISR, and non-DM + ISR) $(P<0.05)$. The proportion of patients with hypertension was highest in the DM + ISR group (85\%). In terms of systolic blood pressure, more patients in the DM + ISR and the DM + non-ISR groups failed to reach the systolic blood pressure control target when compared to the corresponding non-DM + ISR and non-DM + non-ISR groups, though the difference was not statistically significant. In addition, the stent diameter $(2.75 \mathrm{~mm}$ [2.50 $\mathrm{mm} \varangle 3.50 \mathrm{~mm}])$ in the $\mathrm{DM}+\mathrm{ISR}$ group was significantly smaller than that in the other groups (P $<0.05$ ) (Table 2). 
Table 2

Comparison of clinical baseline and coronary lesions in the population

\begin{tabular}{|c|c|c|c|c|c|}
\hline & Non-DM + non-ISR & DM + non-ISR & Non-DM + ISR & $\mathrm{DM}+\mathrm{ISR}$ & $\mathbf{P}$ \\
\hline Age (years) & $63.82 \pm 9.92$ & $65.17 \pm 10.17$ & $64.48 \pm 10.22$ & $65.30 \pm 9.89$ & 0.722 \\
\hline Male, n (\%) & $173((73.62 \%)$ & $49(62.82 \%)$ & $23(85.19 \%)$ & $13(65.00 \%)$ & 0.097 \\
\hline Hypertension, n (\%) & $173((73.62 \%)$ & $62(79.49 \%)$ & $13(48.15 \%)$ & $17(85.00 \%)$ & 0.008 \\
\hline Systolic blood pressure mmHg & $136.38 \pm 21.42$ & $141.29 \pm 20.91$ & $129.26 \pm 22.18$ & $140.95 \pm 17.58$ & 0.055 \\
\hline Diastolic blood pressure, $\mathrm{mmHg}$ & $79.13 \pm 13.17$ & $80.97 \pm 13.04$ & $76.74 \pm 9.84$ & $78.05 \pm 11.60$ & 0.454 \\
\hline Smoking history, n (\%) & $127(54.04 \%)$ & $30(38.46 \%)$ & $18(66.67 \%)$ & $11(55.00 \%)$ & 0.037 \\
\hline Hyperlipidemia, n (\%) & $65(27.66 \%)$ & $26(33.33 \%)$ & $7(25.93 \%)$ & $4(20.00 \%)$ & 0.625 \\
\hline Follow-up interval (months) & $12(1-48)$ & $12(1-37)$ & $13(3-77)$ & $12.5(6-72)$ & 0.022 \\
\hline SAP & 110 & 33 & 9 & 11 & \multirow[t]{2}{*}{0.437} \\
\hline ACS & 125 & 44 & 18 & 9 & \\
\hline LVEF(\%) & $61.56 \pm 6.62$ & $61.63 \pm 7.03$ & $61.04 \pm 8.55$ & $59.43 \pm 9.62$ & 0.692 \\
\hline $\mathrm{LA}(\mathrm{mm})$ & $34.49 \pm 5.10$ & $35.32 \pm 4.58$ & $34.35 \pm 5.25$ & $34.19 \pm 4.72$ & 0.696 \\
\hline Bracket diameter (mm) & $3.00(2.25 \rrbracket 4.00)$ & $3.00(2.25 \rrbracket 4.00)$ & $3.00(1.50 \rrbracket 4.00)$ & $2.75(2.50 \otimes 3.50)$ & 0.026 \\
\hline Bracket length (mm) & $24.00(12.00 \otimes 38.00)$ & $24.00(12.00 \otimes 38.00)$ & $29.00(12.00 \otimes 36.00)$ & $24.00(12.00 \rrbracket 38.00)$ & 0.121 \\
\hline $\begin{array}{l}\text { Maximum balloon inflation } \\
\text { pressure, atm }\end{array}$ & $14.00(8.00 \rrbracket 20.00)$ & $17.00(9.00 \otimes 20.00)$ & $16.00(8.00 \rrbracket 20.00)$ & $16.00(12.00 \otimes 20.00)$ & 0.540 \\
\hline $\begin{array}{l}\text { Average balloon inflation } \\
\text { pressure, atm }\end{array}$ & $15.00(4.00 \rrbracket 26.00)$ & $16.00(9.00 \rrbracket 22.00)$ & $15.00(8.00 \otimes 19.00)$ & $16.00(10.00 \rrbracket 20.00)$ & 0.571 \\
\hline
\end{tabular}

Reexamination of laboratory parameters of pre-PCl and repeat CAG

Table 3 shows that the levels of TC, TG, HDL-c, LDL-c, ApoB100, ApoE, RPL-c, non-HDL-c, TC / HDL-c, TG / HDL-c in the four groups are equivalent pre-PCI and repeat CAG. However, the ApoA1 level in the DM + non-ISR and DM + ISR groups was lower than that in the respective non-DM + non-ISR and non-DM + ISR groups for pre-PCl and repeat CAG. There were statistical differences pre-PCl $(1.00 \pm 0.24 \mathrm{vs}$ $1.09 \pm 0.30, P<0.05 ; 1.01 \pm 0.21$ vs $1.22 \pm 0.52$, in the $D M+$ non-ISR and $D M+$ ISR groups vs the non-DM + non-ISR and non-DM + ISR groups, respectively $P<0.05)$. The DM + non-ISR and DM + ISR groups exhibited lower levels of ApoA1 / ApoB100 compared to the corresponding non-DM + non-ISR and non-DM + ISR groups for pre-PCI and repeat CAG. These differences between the DM-ISR group and the non-DM + ISR group were significant $(P<0.05)$ (pre-PCl $1.35 \pm 0.32$ vs $1.76 \pm 0.86, P<0.05$, repeat CAG $1.62 \pm 0.53$ vs $2.171 .34, P<$ 0.05). The DM + non-ISR and the DM + ISR groups had a higher level of HbA1c, which reflected the blood glucose control level, compared to the corresponding non-DM + non-ISR and non-DM + ISR groups $(P<0.05)$, of which the $D M+I S R$ group demonstrated the highest level; this difference was significant compared with $D M+$ non-ISR group $(P<0.05)$ (pre-PCl $8.15 \pm 1.48$ vs $7.65 \pm 1.66, P<0.05$; repeat CAG $8.12 \pm 1.84$ vs $7.49 \pm 1.38, \mathrm{P}<0.05)$ (Table 3$)$. 
Table 3

Comparison of the laboratory parameters for the four groups

\begin{tabular}{|c|c|c|c|c|c|}
\hline & Non-DM + non-ISR & DM + non-ISR & Non-DM + ISR & $\mathrm{DM}+\mathrm{ISR}$ & $\mathbf{P}$ \\
\hline \multicolumn{6}{|l|}{ Pre-PCl } \\
\hline $\mathrm{TC}, \mathrm{mmol} / \mathrm{L}$ & $4.22 \pm 1.03$ & $4.07 \pm 1.51$ & $3.99 \pm 1.11$ & $3.95 \pm 0.83$ & 0.512 \\
\hline TG,mmol/L & $1.57 \pm 1.01$ & $1.57 \pm 0.79$ & $1.82 \pm 1.55$ & $1.46 \pm 0.99$ & 0.661 \\
\hline HDL-c,mmol/L & $1.10 \pm 0.32$ & $1.00 \pm 0.27$ & $1.10 \pm 0.52$ & $1.08 \pm 0.28$ & 0.119 \\
\hline LDL-c,mmol/L & $2.50 \pm 0.79$ & $2.41 \pm 1.04$ & $2.18 \pm 0.92$ & $2.39 \pm 0.82$ & 0.316 \\
\hline ApoA1,g/L & $1.09 \pm 0.30$ & $1.00 \pm 0.24^{\star}$ & $1.22 \pm 0.52$ & $1.01 \pm 0.21^{\#}$ & 0.015 \\
\hline ApoB100,g/L & $0.81 \pm 0.20$ & $0.81 \pm 0.25$ & $0.74 \pm 0.25$ & $0.80 \pm 0.21$ & 0.511 \\
\hline ApoE,mg/L & $43.08 \pm 17.50$ & $40.76 \pm 16.14$ & $37.86 \pm 15.49$ & $37.08 \pm 12.34$ & 0.254 \\
\hline TC/HDL-c & $4.10 \pm 1.49$ & $4.27 \pm 1.65$ & $3.98 \pm 1.24$ & $3.83 \pm 1.15$ & 0.612 \\
\hline TG/HDL-C & $1.22(0.26 \varangle 9.70)$ & $1.42(0.38 \bowtie 6.39)$ & $1.35(0.51 \llbracket 5.52)$ & $1.09(0.44 \llbracket 6.51)$ & 0.256 \\
\hline LDL-c/HDL-c & $2.44 \pm 0.99$ & $2.55 \pm 1.16$ & $2.20 \pm 0.94$ & $2.44 \pm 1.03$ & 0.473 \\
\hline ApoA1/ ApoB100 & $1.43 \pm 0.54$ & $1.34 \pm 0.54$ & $1.76 \pm 0.86$ & $1.35 \pm 0.32^{\#}$ & 0.028 \\
\hline RPL-c,mmol/L & $0.69 \pm 0.47$ & $0.66 \pm 0.39$ & $0.72 \pm 0.47$ & $0.61 \pm 0.40$ & 0.803 \\
\hline Non-HDL-c,mmol/L & $3.12 \pm 1.01$ & $3.07 \pm 1.48$ & $2.90 \pm 0.96$ & $2.86 \pm 0.79$ & 0.640 \\
\hline $\mathrm{Hcy}, \mu \mathrm{mol} / \mathrm{L}$ & 19.06(2.80ه74.37) & $15.39(6.01 \varangle 94.00)$ & $21.95(9.28 \llbracket 35.06)$ & $14.07(6.67 \otimes 110.70)$ & 0.195 \\
\hline $\mathrm{HbA} 1 \mathrm{c}, \%$ & $5.90 \pm 0.83$ & $7.65 \pm 1.66^{*}$ & $5.76 \pm 1.77$ & $8.15 \pm 1.48^{\# \&}$ & 0.000 \\
\hline UC, $\mu \mathrm{mol} / \mathrm{L}$ & $356.65 \pm 101.45$ & $314.39 \pm 97.09^{*}$ & $328.91 \pm 77.90$ & $295.45 \pm 102.78$ & 0.001 \\
\hline \multicolumn{6}{|l|}{ Repeat CAG } \\
\hline TC,mmol/L & $3.34 \pm 0.88$ & $3.21 \pm 0.88$ & $3.24 \pm 0.79$ & $2.99 \pm 0.69$ & 0.512 \\
\hline TG,mmol/L & $1.38 \pm 0.90$ & $1.42 \pm 0.65$ & $1.30 \pm 0.88$ & $1.25 \pm 0.65$ & 0.856 \\
\hline $\mathrm{HDL}-\mathrm{c}, \mathrm{mmol} / \mathrm{L}$ & $1.06 \pm 0.36$ & $0.97 \pm 0.28$ & $1.06 \pm 0.34$ & $0.97 \pm 0.29$ & 0.244 \\
\hline LDL-c,mmol/L & $1.66 \pm 0.57$ & $1.61 \pm 0.58$ & $1.52 \pm 0.53$ & $1.47 \pm 0.46$ & 0.382 \\
\hline ApoA1,g/L & $1.10 \pm 0.31$ & $1.04 \pm 0.29$ & $1.19 \pm 0.55$ & $0.95 \pm 0.25$ & 0.058 \\
\hline ApoB100,g/L & $0.62 \pm 0.16$ & $0.63 \pm 0.17$ & $0.64 \pm 0.25$ & $0.62 \pm 0.16$ & 0.954 \\
\hline ApoE,mg/L & $32.78 \pm 14.92$ & $31.00 \pm 14.09$ & $29.82 \pm 10.64$ & $31.74 \pm 13.76$ & 0.686 \\
\hline TC/HDL-C & $3.36 \pm 1.21$ & $3.47 \pm 1.11$ & $3.22 \pm 0.99$ & $3.24 \pm 0.83$ & 0.747 \\
\hline TG/HDL-c & $1.12(0.20 \bowtie 17.38)$ & $1.36(0.21 \otimes 5.28)$ & $1.00(0.25 \rrbracket 4.58)$ & $1.13(0.55 \llbracket 4.02)$ & 0.086 \\
\hline LDL-c/HDL-c & $1.69 \pm 0.73$ & $1.75 \pm 0.69$ & $1.55 \pm 0.70$ & $1.69 \pm 0.71$ & 0.660 \\
\hline ApoA1/ ApoB100 & $1.86 \pm 0.64$ & $1.74 \pm 0.57$ & $2.17 \pm 1.34$ & $1.62 \pm 0.53^{\#}$ & 0.033 \\
\hline RPL-c,mmol/L & $0.66 \pm 0.43$ & $0.71 \pm 0.45$ & $0.66 \pm 0.37$ & $0.55 \pm 0.23$ & 0.489 \\
\hline Non-HDL-c,mmol/L & $2.28 \pm 0.83$ & $2.24 \pm 0.82$ & $2.18 \pm 0.77$ & $2.02 \pm 0.58$ & 0.569 \\
\hline $\mathrm{Hcy}, \mu \mathrm{mol} / \mathrm{L}$ & 13.25(3.50冈63.04) & $12.35(5.58 \otimes 40.80)$ & $10.06(6.76 \llbracket 57.87)$ & 13.81(7.21ه33.20) & 0.158 \\
\hline $\mathrm{HbA} 1 \mathrm{c}, \%$ & $5.96 \pm 0.73$ & $7.49 \pm 1.38^{\star}$ & $6.01 \pm 0.65$ & $8.12 \pm 1.84^{\# \&}$ & 0.000 \\
\hline UC, $\mu \mathrm{mol} / \mathrm{L}$ & $363.04 \pm 97.87$ & $334.47 \pm 96.36$ & $337.35 \pm 85.45$ & $337.93 \pm 95.72$ & 0.105 \\
\hline
\end{tabular}


All four groups showed a decrease in the levels of TC, TG, LDL-C, ApoB100, and ApoE level at repeat CAG compared with pre-PCl, which indicated that conventional statin drug therapy after PCl exhibited a certain effect, but that HDL-c and ApoA1 prohibited the difference in performance. The HDL-c level in the DM + ISR group showed a significant downward trend, while the other three groups maintained the pre$P C l$ level. At repeat $C A G$, the ApoA1 level in the DM + ISR group was lower than that pre-PCl, while the other three groups had higher levels of ApoA1 than at pre-PCl. The HbA1c level in the non-DM + ISR group showed a significant increase, while in the other three groups it remained unchanged, suggesting that there may be patients with an abnormal glucose tolerance in the non-DM + ISR group. The uric acid level in the DM + ISR group showed a remarkably increased trend, which was significantly larger than the other three groups, but it did not reach statistical significance (Table 4).

Table 4

Comparison of the changes of laboratory parameters between the four groups at pre-PCl and repeat CAG

\begin{tabular}{|c|c|c|c|c|c|}
\hline & Non-DM + non-ISR & DM + non-ISR & Non-DM + ISR & DM + ISR & $\mathbf{P}$ \\
\hline$\triangle \mathrm{TC}, \%$ & $-18.72 \pm 21.11$ & $-15.51 \pm 32.62$ & $-16.89 \pm 22.00$ & $-22.84 \pm 21.69$ & 0.628 \\
\hline$\triangle T G, \%$ & $-0.56 \pm 53.66$ & $-2.41 \pm 45.30$ & $-11.53 \pm 43.24$ & $3.51 \pm 46.61$ & 0.779 \\
\hline$\triangle \mathrm{HDL}-\mathrm{c}, \%$ & $0.88 \pm 30.69$ & $-0.07 \pm 22.69$ & $-0.24 \pm 20.00$ & $-10.34 \pm 21.08$ & 0.425 \\
\hline$\triangle \mathrm{LDL}-\mathrm{C}, \%$ & $-29.84 \pm 25.85$ & $-26.24 \pm 34.12$ & $-27.63 \pm 24.21$ & $-32.98 \pm 33.70$ & 0.730 \\
\hline$\triangle \mathrm{ApoA} 1, \%$ & $6.96 \pm 39.78$ & $8.32 \pm 33.29$ & $6.26 \pm 59.55$ & $-3.67 \pm 27.00$ & 0.694 \\
\hline$\triangle \mathrm{ApoB} 100, \%$ & $-20.71 \pm 22.57$ & $-18.24 \pm 27.37$ & $-10.74 \pm 27.02$ & $-15.70 \pm 27.54$ & 0.295 \\
\hline$\triangle \mathrm{ApoE}, \%$ & $-17.96 \pm 36.55$ & $-19.55 \pm 30.10$ & $-11.70 \pm 36.19$ & $-7.53 \pm 44.04$ & 0.519 \\
\hline$\triangle \mathrm{TC} / \mathrm{HDL}-\mathrm{C}, \%$ & $-15.40 \pm 27.24$ & $-13.13 \pm 36.27$ & $-14.11 \pm 23.75$ & $-11.96 \pm 23.11$ & 0.918 \\
\hline$\triangle \mathrm{TG} / \mathrm{HDL}-\mathrm{c}, \%$ & $8.22 \pm 77.77$ & $2.87 \pm 54.09$ & $-10.41 \pm 44.40$ & $19.99 \pm 57.38$ & 0.526 \\
\hline $\begin{array}{l}\triangle \mathrm{LDL}-\mathrm{C} / \mathrm{HDL}-\mathrm{C}, \\
\%\end{array}$ & $-26.28 \pm 32.22$ & $-23.16 \pm 42.39$ & $-22.58 \pm 36.22$ & $-22.60 \pm 39.79$ & 0.730 \\
\hline$\triangle \mathrm{ApoA} 1 / \mathrm{ApoB} 100, \%$ & $44.66 \pm 66.02$ & $45.58 \pm 60.43$ & $37.25 \pm 102.55$ & $28.28 \pm 56.09$ & 0.734 \\
\hline$\triangle \mathrm{RPL}-\mathrm{c}, \mathrm{mmol} / \mathrm{L}$ & $-0.03 \pm 0.39$ & $-0.06 \pm 0.52$ & $-0.03 \pm 0.48$ & $-0.03 \pm 0.47$ & 0.572 \\
\hline $\begin{array}{l}\triangle \text { Non-HDL-c, } \\
\mathrm{mmol} / \mathrm{L}\end{array}$ & $-0.86 \pm 0.92$ & $-0.90 \pm 1.56$ & $-0.75 \pm 0.98$ & $-0.88 \pm 0.95$ & 0.960 \\
\hline$\triangle \mathrm{Hcy}, \mu \mathrm{mol} / \mathrm{L}$ & $-4.10 \pm 012.58$ & $-3.76 \pm 13.82$ & $-5.93 \pm 15.69$ & $-7.07 \pm 24.08$ & 0.859 \\
\hline$\triangle \mathrm{HbA} 1 \mathrm{c}, \%$ & $0.03 \pm 0.56$ & $-0.20 \pm 1.88$ & $0.42 \pm 1.84$ & $-0.13 \pm 1.51$ & 0.382 \\
\hline$\triangle \mathrm{UC}, \mu \mathrm{mol} / \mathrm{L}$ & $10.55 \pm 82.34$ & $16.80 \pm 62.85$ & $-0.17 \pm 56.27$ & $41.11 \pm 99.94$ & 0.191 \\
\hline
\end{tabular}

Data were expressed as mean \pm SD

Note: TC, total cholesterol; TG, triglyceride; HDL-c, high-density lipoprotein cholesterol; LDL-c, low-density lipoprotein cholesterol; ApoA1, apolipoprotein A1; ApoB100, apolipoprotein B100; ApoE, contained Lipoprotein E; RPL-C, residual lipoprotein; Non-HDL-C, non-highdensity lipoprotein cholesterol; Hcy, homocysteine; HbA1c, glycated haemoglobin A1c; UC, uric acid.

We further compared the changes of blood lipid composition of the DM + non-ISR and the DM + ISR groups for the pre-PCI and repeat CAG at follow-up. The TC, LDL-c, ApoB100, ApoE, non-HDL-c, TC / HDL-C, LDL-c / HDL-c levels showed a downward trend, and for both groups, the above indicators were statistically different at pre-PCl and repeat $C A G(P<0.05)$. The decline in the levels of TG and TG / HDL-c in the $D M+$ non-ISR group was greater than those of the DM + ISR group. The HDL-c and ApoA1 levels in the DM + ISR group showed a downward trend, while in the DM + non-ISR group these two indictors remained unchanged or were slightly increased. Similarly, the increase in the levels of ApoA1 / ApoB100 in the DM + non-ISR group was greater than those in the DM + ISR group (Fig. 1,2).

\section{Gensini Points And Progress}

Table 5 reveals that in the DM + ISR and DM + non-ISR groups, the Gensini score and the detaching stent Gensini score (no-stent) at the time of $\mathrm{PCl}$ and repeat CAG were higher than those of the non-DM + non-ISR and Non-DM + ISR groups $(P<0.05)$. Regarding the Gensini 
progress, the DM + ISR and DM + non-ISR groups showed more progress compared with the non-DM groups, but did not reach statistical significance.

Table 5

Four $\mathrm{PCl}$ groups and a review of the CAG Gensini score and progress

\begin{tabular}{|c|c|c|c|c|c|}
\hline & Non-DM + non-ISR & DM + non-ISR & Non-DM + ISR & $\mathrm{DM}+\mathrm{ISR}$ & $\mathbf{P}$ \\
\hline \multicolumn{6}{|l|}{$\mathrm{PCl}$} \\
\hline $\begin{array}{l}\text { Gensini } \\
\text { score }\end{array}$ & $48.50(8.00 \otimes 196.00)$ & $54.00(10.00 \bigotimes 150.00)$ & $38.00(4.00 \otimes 136.00)$ & $52.00(13.00 \otimes 96.00)$ & 0.082 \\
\hline Ln(Gensini) & $3.81 \pm 0.64$ & $4.01 \pm 0.61^{\star}$ & $3.63 \pm 0.89$ & $3.87 \pm 0.57$ & 0.040 \\
\hline $\begin{array}{l}\text { Gensini } \\
\text { (no-stent) }\end{array}$ & $12.00(0.00 \rrbracket 80.00)$ & $19.00(0.00 \otimes 133.00)$ & $10.00(0.00 \otimes 56.00)$ & $24.00(0.00 \otimes 53.00)$ & 0.015 \\
\hline $\begin{array}{l}\text { LnGensini } \\
\text { (non-stent) }\end{array}$ & $2.62 \pm 0.95$ & $2.84 \pm 0.99$ & $2.52 \pm 1.04$ & $3.00 \pm 0.71$ & 0.133 \\
\hline \multicolumn{6}{|l|}{$\begin{array}{l}\text { Repeat } \\
\text { CAG }\end{array}$} \\
\hline $\begin{array}{l}\text { Gensini } \\
\text { (no-stent) }\end{array}$ & $13.00(0.00 \otimes 85.00)$ & 21.00(0.00ه140.00) & $10.00(0.00 \bowtie 68.00)$ & $25.50(0.00 \otimes 74.00)$ & 0.002 \\
\hline $\begin{array}{l}\text { LnGensini } \\
\text { (non-stent) }\end{array}$ & $2.68 \pm 0.95$ & $2.90 \pm 1.06$ & $2.45 \pm 1.00$ & $3.07 \pm 0.67$ & 0.069 \\
\hline $\begin{array}{l}\triangle \text { Gensini } \\
\text { (no-stent) }\end{array}$ & $1.32 \pm 17.00$ & $4.42 \pm 21.44$ & $0.91 \pm 14.82$ & $2.90 \pm 14.28$ & 0.598 \\
\hline \multicolumn{6}{|c|}{ Data were expressed as mean $\pm S D$, median with minimum and maximum } \\
\hline Note: Compa & d with non-DM + non & $I S R * P<0.05$ & & & \\
\hline
\end{tabular}

\section{Changes of the inflammation index for all patients at pre-PCl and later repeat CAG}

Table 6 shows that there are no significant differences in CRP, WBC, L, M, Hb, PLT, PDW, RDW, PLT / L, and M / HDL-c in the four groups pre$\mathrm{PCl}$. However, at repeat CAG, the CRP, WBC, and $\mathrm{N}$ levels in the DM + non-ISR and DM + ISR groups were higher than those in the corresponding non-DM + non-ISR and non-DM + ISR groups. Considering the above three indexes, the DM + ISR group had the highest values compared with the other three groups $(\mathrm{P}<0.05)$. Further exploration of the changes in the inflammation index revealed that the $\mathrm{DM}$ + ISR group prohibited an increasing trend in the WBC and $\mathrm{N}$ values(WBC, pre-PCI 6.86 \pm 2.37, repeat CAG 7.07 \pm 2.63 ; $\mathrm{N}$, pre-PCI 4.55 \pm 2.05 ,repeat CAG $4.93 \pm 2.50$ ) while the other three groups showed a downward trend. There were statistical differences between the DM + ISR and the $\mathrm{DM}+$ non-ISR groups $(\triangle \mathrm{WBC} 0.20 \pm 1.78$ vs $1.91 \pm 3.25, \mathrm{P}<0.05 ; \triangle \mathrm{N} 0.41 \pm 1.54$ vs $1.84 \pm 3.28, \mathrm{P}<0.05)($ Table 7$)$. Figure 3 shows more intuitively that the levels of WBC, N, N / L, and CRP in the DM + ISR group present an increasing trend at repeat CAG compared to pre-PCI. However, the levels of WBC, N, N / L, and CRP in the DM + non-ISR group decreased significantly, and there was a statistically significant difference between the index at pre-PCl and at repeat $\mathrm{CAG}(\mathrm{P}<0.05)$. (Fig. 3a-d). 
Table 6

Inflammation indexes in the four groups at pre-PCI and repeat CAG

\begin{tabular}{|c|c|c|c|c|c|}
\hline & Non-DM + non-ISR & DM + non-ISR & Non-DM + ISR & $\mathrm{DM}+\mathrm{ISR}$ & $\mathbf{P}$ \\
\hline \multicolumn{6}{|l|}{ Pre-PCl } \\
\hline CRP,mg/L & $1.64(0.00 \otimes 110.13)$ & $2.03(0.50 \otimes 100.87)$ & $2.11(0.00 \otimes 22.00)$ & $3.15(0.00 \bigotimes 32.18)$ & 0.573 \\
\hline WBC, $10^{\wedge} 9 / \mathrm{L}$ & $7.66 \pm 3.28$ & $8.02 \pm 3.67$ & $6.37 \pm 2.52$ & $6.86 \pm 2.37$ & 0.109 \\
\hline$L, 10^{\wedge} 9 / \mathrm{L}$ & $1.50 \pm 0.59$ & $1.62 \pm 0.75$ & $1.35 \pm 0.51$ & $1.73 \pm 0.90$ & 0.109 \\
\hline $\mathrm{N}, 10^{\wedge} 9 / \mathrm{L}$ & $5.65 \pm 3.38$ & $5.86 \pm 3.37$ & $4.46 \pm 2.38$ & $4.55 \pm 2.05$ & 0.121 \\
\hline $\mathrm{M}, 10^{\wedge} 9 / \mathrm{L}$ & $0.46 \pm 0.26$ & $0.48 \pm 0.40$ & $0.44 \pm 0.23$ & $0.45 \pm 0.19$ & 0.940 \\
\hline $\mathrm{Hb}, \mathrm{g} / \mathrm{L}$ & $134.65 \pm 17.37$ & $133.42 \pm 15.78$ & $133.37 \pm 15.44$ & $135.05 \pm 22.63$ & 0.938 \\
\hline $\mathrm{PLT}, 10^{\wedge} 9 / \mathrm{L}$ & $190.26 \pm 59.20$ & $175.73 \pm 49.36$ & $170.00 \pm 43.57$ & $181.60 \pm 55.94$ & 0.099 \\
\hline $\mathrm{RDW}, \%$ & $13.09 \pm 0.90$ & $13.47 \pm 2.00$ & $13.07 \pm 0.63$ & $13.33 \pm 0.78$ & 0.103 \\
\hline PDW,\% & $15.16 \pm 2.72$ & $15.58 \pm 2.38$ & $16.23 \pm 2.94$ & $15.12 \pm 2.26$ & 0.180 \\
\hline $\mathrm{N} / \mathrm{L}$ & $3.17(0.76 \rrbracket 28.14)$ & $2.93(1.10 \rrbracket 27.64)$ & $3.13(0.76 \bigotimes 11.01)$ & $2.79(0.92 \otimes 13.50)$ & 0.509 \\
\hline PLT/L & $144.60 \pm 73.44$ & $128.24 \pm 63.28$ & $138.54 \pm 51.02$ & $133.06 \pm 79.75$ & 0.334 \\
\hline $\mathrm{M} / \mathrm{HDL}-\mathrm{C}$ & $0.46 \pm 0.32$ & $0.52 \pm 0.47$ & $0.45 \pm 0.26$ & $0.45 \pm 0.22$ & 0.592 \\
\hline \multicolumn{6}{|l|}{ Repeat CAG } \\
\hline CRP,mg/L & $0.75(0.50 \otimes 186.00)$ & $0.97(0.50 \rrbracket 42.95)$ & $1.37(0.50 \otimes 70.80)$ & $2.33(0.50 \rrbracket 48.02)$ & 0.009 \\
\hline WBC, $10^{\wedge} 9 / \mathrm{L}$ & $5.86 \pm 1.50$ & $6.21 \pm 1.69$ & $5.60 \pm 2.01$ & $7.07 \pm 2.63^{\star \# \&}$ & 0.008 \\
\hline $\mathrm{L}, 10^{\wedge} 9 / \mathrm{L}$ & $1.52 \pm 0.73$ & $1.48 \pm 0.56$ & $1.32 \pm 0.43$ & $1.32 \pm 0.43$ & 0.572 \\
\hline $\mathrm{N}, 10^{\wedge} 9 / \mathrm{L}$ & $3.79 \pm 1.21$ & $4.14 \pm 1.33$ & $3.69 \pm 1.71$ & $4.93 \pm 2.50^{\star \# \&}$ & 0.002 \\
\hline $\mathrm{M}, 10^{\wedge} 9 / \mathrm{L}$ & $0.42 \pm 0.17$ & $0.41 \pm 0.20$ & $0.43 \pm 0.18$ & $0.46 \pm 0.20$ & 0.756 \\
\hline $\mathrm{Hb}, \mathrm{g} / \mathrm{L}$ & $136.35 \pm 16.89$ & $132.96 \pm 15.46$ & $133.48 \pm 15.46$ & $130.79 \pm 18.75$ & 0.266 \\
\hline $\mathrm{PLT}, 10^{\wedge} 9 / \mathrm{L}$ & $175.90 \pm 55.64$ & $163.37 \pm 51.74$ & $160.68 \pm 55.74$ & $189.58 \pm 55.89$ & 0.121 \\
\hline RDW,\% & $13.26 \pm 0.90$ & $13.51 \pm 1.00$ & $13.42 \pm 1.07$ & $13.33 \pm 1.18$ & 0.262 \\
\hline PDW,\% & $13.26 \pm 0.90$ & $13.51 \pm 1.00$ & $13.42 \pm 1.07$ & $13.33 \pm 1.18$ & 0.262 \\
\hline$N / L$ & $2.58(0.71 \otimes 16.00)$ & $2.86(1.07 \rrbracket 8.63)$ & $2.56(1.40 \rrbracket 7.62)$ & $3.17(1.53 \rrbracket 11.84)$ & 0.175 \\
\hline PLT/L & $128.89 \pm 54.08$ & $122.19 \pm 49.84$ & $131.07 \pm 56.10$ & $146.67 \pm 78.18$ & 0.377 \\
\hline M/HDL-c & $0.44 \pm 0.23$ & $0.47 \pm 0.28$ & $0.43 \pm 0.19$ & $0.55 \pm 0.33$ & 0.311 \\
\hline
\end{tabular}


Table 7

The changes of inflammation indicators in the four groups between pre-PCl and re-examination of CAG

\begin{tabular}{|c|c|c|c|c|c|}
\hline & Non-DM + non-ISR & DM + non-ISR & Non-DM + ISR & DM + ISR & $\mathbf{P}$ \\
\hline$\triangle \mathrm{CRP}, \mathrm{mg} / \mathrm{L}$ & $-3.97 \pm 22.13$ & $-3.93 \pm 16.79$ & $0.38 \pm 12.11$ & $1.14 \pm 13.60$ & 0.564 \\
\hline$\triangle \mathrm{WBC}, 10^{\wedge} 9 / \mathrm{L}$ & $-1.76 \pm 2.80$ & $-1.91 \pm 3.25$ & $-0.83 \pm 2.41$ & $0.20 \pm 1.78^{\star \#}$ & 0.013 \\
\hline$\triangle \mathrm{L}, 10^{\wedge} 9 / \mathrm{L}$ & $-0.02 \pm 0.66$ & $-0.13 \pm 0.58$ & $0.02 \pm 0.49$ & $-0.23 \pm 0.92$ & 0.178 \\
\hline$\triangle \mathrm{N}, 10^{\wedge} 9 / \mathrm{L}$ & $-1.85 \pm 3.06$ & $-1.84 \pm 3.28$ & $-0.85 \pm 2.58$ & $0.41 \pm 1.54^{\star \#}$ & 0.009 \\
\hline$\triangle \mathrm{M}, 10^{\wedge} 9 / \mathrm{L}$ & $-0.04 \pm 0.26$ & $-0.07 \pm 0.33$ & $-0.02 \pm 0.23$ & $0.00 \pm 0.23$ & 0.653 \\
\hline$\triangle \mathrm{Hb}, \mathrm{g} / \mathrm{L}$ & $1.14 \pm 19.76$ & $-0.11 \pm 14.21$ & $-0.08 \pm 11.97$ & $-1.95 \pm 14.93$ & 0.867 \\
\hline$\triangle \mathrm{PLT}, 10^{\wedge} 9 / \mathrm{L}$ & $-14.86 \pm 44.50$ & $-12.95 \pm 35.55$ & $-11.12 \pm 38.19$ & $-5.05 \pm 43.96$ & 0.270 \\
\hline$\triangle \mathrm{RDW}$ & $0.18 \pm 1.01$ & $0.02 \pm 2.14$ & $0.38 \pm 0.99$ & $-0.02 \pm 0.86$ & 0.611 \\
\hline$\triangle \mathrm{PDW}$ & $1.11 \pm 3.65$ & $0.74 \pm 2.55$ & $0.27 \pm 3.00$ & $0.34 \pm 2.59$ & 0.492 \\
\hline$\triangle N / L$ & $-1.82 \pm 4.55$ & $-1.58 \pm 4.35$ & $-0.67 \pm 2.73$ & $0.29 \pm 1.79$ & 0.149 \\
\hline$\triangle \mathrm{PLT} / \mathrm{L}$ & $-14.84 \pm 74.90$ & $-7.72 \pm 58.75$ & $-9.11 \pm 59.00$ & $11.02 \pm 53.17$ & 0.436 \\
\hline$\triangle M / H D L-C$ & $-0.03 \pm 0.31$ & $-0.07 \pm 0.39$ & $-0.01 \pm 0.29$ & $0.09 \pm 0.30$ & 0.298 \\
\hline
\end{tabular}

Data were expressed as mean \pm SD

Note: CRP, C-reactive protein; WBC, white blood cells; L, lymphocytes; M, monocytes; Hg, haemoglobin; PLT, platelet values; PDW, platelet width; RDW, red blood cell width; PLT / N, platelets / lymphocytes Ratio; M / HDL-c monocytes / high-density lipoprotein cholesterol. Compared with non-DM + non-ISR * $\mathrm{P}<0.05$, compared with non-DM + ISR ${ }^{\#} \mathrm{P}<0.05$, compared with $\mathrm{DM}+$ non- ISR comparison \& $\mathrm{P}<$ 0.05 .

\section{Correlation of risk factors with the changes of the coronary Gensini score in DM}

The Gensini score (no-stent) was calculated for the DM + non-ISR and DM + ISR groups at pre-PCI period and repeat CAG, and correlation analysis was used to evaluate the risk factors and the Gensini score change. We found that levels of TG / HDL-c at the pre-PCI period and TG, WBC, N level, LDL-c / HDL-c, N / L, TG / HDL-c at the repeat CAG period were positively correlated with the $\triangle$ Gensini, with correlation coefficients of $0.210,0.239,0.229,0.315,0.314,0.327$, and 0.349 , respectively. Furthermore, $\triangle$ Gensini was negatively correlated with HDL-C, and $\mathrm{ApoA} 1$ levels at the pre-PCI period, and the HDL-c level at the repeat CAG period, with correlation coefficients of -0.233 , -0.227 , and -0.0.301, respectively (Fig. 4 a-j).

\section{Analysis of multiple risk factors of DM-ISR with the cumulative incidence of ISR Kaplan-Meier plot in the DM and non-DM groups}

Binary logistics regression analysis was used to analyse the risk factors of ISR in patients with DM. Single factor logistics regression analysis showed that $\triangle \mathrm{WBC}$ and $\triangle \mathrm{N}$ were identified as risk factors $(\triangle \mathrm{WBC}, \mathrm{OR} 1.466,95 \% \mathrm{Cl} 1.079-1.993 ; \triangle \mathrm{N}$ OR $1.612,95 \% \mathrm{Cl} 1.125-$ 2.310), but in the multivariate regression analysis model, $\triangle \mathrm{N}$ and $\mathrm{HbA} 1 \mathrm{c}-\mathrm{B}$ were revealed as risk factors, $(\triangle \mathrm{N}, \mathrm{OR} 1.929,95 \% \mathrm{Cl} 1.216-$ 3.058; HbA1c-B OR 1.559,95\% Cl 1.001-1.707) (Table 8). According to ROC, the cut-off values were: $\triangle \mathrm{N}$ : $-0.43 * 10 \wedge 9$ / L; $\triangle$ WBC: -0.565 * $10^{\wedge} 9 / \mathrm{L} ; \mathrm{HbA} 1 \mathrm{c}-\mathrm{B} 7.75 \%$. Based on the independent predictive factors screened from the logistic regression analysis, we incorporated these factors into the restenosis risk prediction model $(P=[\exp (-4.842+0.562(\triangle N)+0.473(H b A 1 c 1-B))] /[1+\exp (-4.842+0.562(\triangle N)$ $+0.473(\mathrm{HbA} 1 \mathrm{c} 1-\mathrm{B}))$ ], to test whether this model was able to predict DES-ISR risk. We used the ROC curve to confirm that $\triangle \mathrm{N}, \triangle \mathrm{WBC}$, $\mathrm{HbA1c}-\mathrm{B}$, and this model were predictive of DES-ISR, then we observed that the restenosis risk prediction model presented a highly predictive value for raised restenosis risk $(\triangle \mathrm{N}, \mathrm{AUC} 0.735,95 \% \mathrm{Cl} 0.603-0.858 ; \triangle \mathrm{WBC}, \mathrm{AUC} 0.682,95 \% \mathrm{Cl} 0.535-0.828 ; \mathrm{HbA} 1 \mathrm{C}-\mathrm{B}, \mathrm{AUC}$ 0.609, 95\% $\mathrm{Cl} 0.453-0.765)$; and for the restenosis risk prediction model for restenosis risk, AUC 0.808, 95\% Cl 0.705-0.912 (Fig. 5a). The cumulative incidence of ISR in the DM group was $20.41 \%$, which was higher than that in the non-diabetic group at $10.31 \%$, (HR 2.369 [95\% Cl (1.231-4.557)]) (Fig. 5b). 
Table 8

Univariate and multivariate logistic regression analysis of predictors of stent restenosis in diabetic patients

\begin{tabular}{|c|c|c|c|c|}
\hline & Univariable Analysis & & Multivariable Analysis & \\
\hline Variables & OR(95\%Cl) & $\mathrm{P}$ & OR(95\%Cl) & $\mathrm{P}$ \\
\hline ApoA1-B/ApoB100-B & $1.008(0.368-2.755)$ & 0.998 & & \\
\hline ApoA1-F/ApoB100-F & 0.998(0.993-1.003) & 0.410 & & \\
\hline $\mathrm{HbA1c-B}$ & $1.196(0.871-1.633)$ & 0.258 & $1.559(1,001-2.428)$ & 0.050 \\
\hline $\mathrm{HbA1c-F}$ & $1.287(0.930-1.780)$ & 0.129 & & \\
\hline WBC-F & $1.233(0.960-1.584)$ & 0.101 & & \\
\hline $\mathrm{N}-\mathrm{F}$ & $1.293(0.964-1.735)$ & 0.086 & & \\
\hline$\triangle W B C$ & $1.466(1.079-1.993)$ & 0.014 & & \\
\hline$\Delta \mathbf{N}$ & $1.612(1.125-2.310)$ & 0.009 & $1.929(1.216-3.058)$ & 0.005 \\
\hline
\end{tabular}

\section{Relationship between LDL-c control level and Gensini score progress in patients with DM}

According to whether the LDL-c value was less than $1.8 \mathrm{mmol} / \mathrm{L}$ at repeat CAG or was decreased by more than $50 \%$ compared with pre-PCl, all patients were assigned to the LDL-c achieved group or the LDL-c failure group. The coronary artery Gensini scores of the two groups $(\mathrm{DM}+$ non-ISR, and DM + ISR) at the pre-PCI period were similar, with no statistical difference (LDL-C achieved group 60.86 $\pm 35.11 ; \mathrm{LDL}-\mathrm{C}$ failure group $68.59 \pm 34.82$ ) (Fig. 6a). then the Gensini score (no-stent) was calculated for both groups at pre-PCl and at repeat CAG, respectively. The LDL-c failure group showed a remarkable increase in the Gensini score (no-stent) [pre-PCl, 20 (6-144); repeat CG, 27 (2$140), P<0.05]$. The $\triangle$ Gensini score for the LDL-c achieved group was significantly reduced compared to the LDL-c failure group, $(\triangle G e n s i n i$ score, LDL-c achieved group, $0.56 \pm 12.08$; LDL-c failure group, $11.87 \pm 31.18, \mathrm{P}<0.05)$ (Fig. 6b, c).

\section{Discussion}

Our study revealed that patients with DM were susceptible to an occurrence of ISR. The reduction of HDL-c and ApoA1 levels, the increase of inflammation indicators (leukocytes and neutrophils) and HbA1c levels were possible risk factors for the occurrence of ISR in patients with DM. The progression of coronary stenosis was more obvious in patients with DM in which the LDL-c level failed to achieve the control target. Our findings indicated that patients with coronary artery disease who also had DM were at a high risk for an occurrence of ISR if they had preoperative risk factors including dyslipidaemia, elevated inflammatory factors, and a high Gensini score. Our results also showed that dynamic observation of the changes of preoperative and postoperative comprehensive risk factors was helpful to identify ISR in patients with DM.

Patients with DM are 2-4 times more likely to suffer from cardiovascular disease than patients without DM[7]. Additionally, cardiovascular disease accounts for $3 / 4$ of the causes of death in patients with DM. Notably, DM itself can lead to a greater inflammatory response, and a more diffuse and rapid progression of atherosclerosis [7]. The ISR occurrence rate in patients with DM is $10 \%$ higher than that in patients without DM [14-16]. Our study found that ISR accounted for 20/98 vs $27 / 262$ in DM and non-DM populations respectively $(P<0.05)$, which was consistent with previous studies.

DM inhibits the ability of vascular endothelial cells to secrete vasodilators such as prostaglandin $\mathrm{I}_{2}\left(\mathrm{PGI}_{2}\right)$ and nitric oxide (NO), while the secretion of vasoconstrictor endothelin-1 increases, which causes vasodilation disorders and vascular sclerosis [8]. In addition, insulin can directly promote the release of platelet-derived NO, activate platelet-derived cyclic guanosine monophosphate, and inhibit platelet aggregation. In addition, platelet aggregation is regulated by endothelial-derived $\mathrm{PGI}_{2}$ and $\mathrm{NO}$, As a result, DM could reduce the secretion of endothelial-derived $\mathrm{PGI}_{2}$ and $\mathrm{NO}$, which are secreted by the vascular endothelium, to ultimately induce platelet aggregation.

Hyperglycaemia causes increased synthesis of thromboxane $A_{2}$, which further leads to vasoconstriction and platelet aggregation [17]. Also, due to the activation of partial coagulation factors $\varangle$, and $\otimes$, tissue factors, and Von Willibrand factors, patients with DM are in a hypercoagulable state, in which they are prone to form thrombosis, which can lead to ischaemia [8]. 
In addition, multiple cross-sectional studies have shown that the levels of inflammatory factors such as CRP, interleukin-6, and tumour necrosis factor-a in patients with DM or in patients with insulin resistance are higher than those in the control group [18], and drugs such as rosiglitazone and pioglitazone can reduce CRP levels [19]. DM could also increase vascular extracellular matrix degradation-related enzyme (matrix metalloprotease-2, matrix metalloprotease-9) levels, resulting in vascular remodelling [20]. Consequently, the majority of the vascular lesions caused by DM are diffuse, and the lack of compensation for the blood vessels leads to a high incidence of cardiovascular events. Therefore, DM can result in vascular lesions arising from multiple mechanisms such as vasodilation disorder, platelet accumulation, thrombosis, inflammation, vascular remodelling, among others. Intensive blood glucose control can reduce the occurrence of cardiovascular events in type $1 \mathrm{DM}$, but no significant benefit was obtained in people with type $2 \mathrm{DM}$ with an $\mathrm{HbA} 1 \mathrm{c}$ level $\leq$ $7.0 \%[21,22]$. Further research found that in the BMS implanted population, HbA1c levels were negatively correlated with BMS-ISR, but were not significantly correlated with DES-ISR [23]. This finding is inconsistent with our results.

Approximately $67 \%$ of patients with DM have dyslipidaemia, most of whom have an abnormally elevated level of VLDL-c and triglycerides and a low HDL-c level, while the level of LDL-c is mostly normal [24]. In patients with DM with an VLDL-c $\geq 0.52 \mathrm{mmol} / \mathrm{L}$, the HR of the VLDL-c and ISR reaches 3.01; thus, the VLDL-c can be used as an independent risk factor for ISR. The mechanism is that DM is complicated by insulin resistance, which causes an excessive production of VLDL-c in the liver, which decreases the ability to remove lipids from the blood after meals. Also, each VLDL-c can transfer more cholesterol than LDL-C; consequently, VLDL-c is engulfed by vascular wall macrophages, which induces inflammation and atherosclerosis, and the resulting continuous inflammatory stimulation and cell proliferation eventually lead to DM-ISR $[25,26]$. Our study found that at the pre-PCI and repeat CAG periods the values of CAG TC, TG, HDLc, LDL-c, ApoB100, ApoE, RPL-c, non-HDL-c, TC/HDL-c, and TG / HDL-c in the DM non-ISR and DM + ISR groups were roughly equivalent, and revealed no statistical differences. However, the levels of ApoA1 in the DM + non-ISR and in the DM + ISR groups were lower than those in the non-DM + non-ISR and non-DM + ISR groups at the pre-PCI and repeat CAG periods, The DM + ISR group had the highest level of $\mathrm{HbA1c}$ and the most significant coronary stenosis Gensini score progression, indicating that this group had the worst blood glucose control, indirectly demonstrated that the level of blood glucosein control in DM can significantly influence the progression of disease and the occurrence of ISR. In addition, the changes of the inflammation indicators such as WBCs and neutrophils in the DM + ISR group were the most obvious, suggesting that the inflammatory response was involved in the process of DM-related ISR. Multivariate regression analysis also concluded that the glycated haemoglobin and neutrophil change values were independent risk factors for ISR.

This study includes some limitations. This study did not employ randomisation; therefore, there was a possibility for selection bias. Also this study was conducted as a single centre with DES implantation patients. A future study with an increased number of patients is required to observe whether there are statistical differences. This study could only identify the association between clinical risk factors and restenosis, it could not confirm a causal relationship. The ISR definition used in this study was based on the independent evaluation of CAG images by two interventional cardiologists. Our study did not use intravascular ultrasound or optical computed tomography to assess the degree of in-stent re-narrowing. Most of the previous relevant studies have focused on predicting ISR through the pre-PCI baseline risk factor level.

Despite these limitations, our study focused on the comprehensive study of ISR in patients with DM with regard to blood lipid, blood glucose and inflammation control levels, which has not been extensively studied.

\section{Conclusions}

In conclusion, this study focus on the dynamic change of lipid and inflammatory indexes, rarely in previous researches. We concluded that coronary artery disease patients with DM had a high risk for ISR if they had preoperative risk factors including dyslipidaemia, elevated inflammatory factors, and a high Gensini score. Dynamic observation of the changes of the preoperative and postoperative comprehensive risk factors is helpful to identify ISR in patients with DM.

\section{Abbreviations}

ApoA1: Apolipoprotein

ApoB100: Apolipoprotein B100

ApoE: Apolipoprotein E

AUC: Area under the curve

BMS: Bare metal stents 
CAG: coronary angiography

Ccr: creatinine

CHD: Coronary heart disease

CRP, C-reactive protein

DES: Drug-eluting stent

DM, Diabetes Mellitus

Hb: Haemoglobin

HbA1c: Glycated haemoglobin

Hcy: Homocysteine

HDL-c: High-density lipoprotein cholesterol

HR: Hazard ratio

ISR: In-stent restenosis

IVUS: intravascular ultrasonography

L: Lymphocyte

LA: Left atrium

LDL-c: Low-density lipoprotein

LVEF: left ventricular ejection

M: Monocytes

MI: Myocardial infarction

$\mathrm{N}$ : Neutrophil

NO: Nitric oxide

PCl: Percutaneous coronary intervention

PDW: Platelet distribution width

PGI2: Prostaglandin I2

PLT: platelet value

RBC: Red blood cell

RDW: RBC distribution width

ROC: Receiver operating characteristic curve

RPL-C: Residual lipoprotein

TC: Total cholesterol

TG: Triglyceride

TIMI: thrombolysis in myocardial infarction 
TLR: Target lesion revascularisation

UC: Uric acid

WBC: White blood cell

\section{Declarations}

\section{Acknowledgements}

The authors thank all the staff and participants of this study for their important contributions.

\section{Authors' contributions}

Z-SG and H-BJ completed the project, analyzed the data, and drafted the manuscript. H-BJ designed the study, interpreted the data and contributed to critically revising the manuscript. J-LQ interpreted the data and contributed to revising the manuscript. X-JJ, C-ZL, T-AQ, J-L, H-JB, Z-B, Z-ZX, S-FJ, Z-XY, Z-R,J-FF contributed to recruitment of patients and clinical diagnosis of disease. All authors read and approved the final manuscript.

\section{Funding}

This work was funded by ZheJiang Provincial Natural Science Foundation of China (LQ19H020003), the ZheJiang Provincial Medical Health Science and Technology Program Foundation (2019327710), Zhejiang Traditional Chinese Medicine Administration(2015ZA204) and the Science Technology Department of JiaXing (2016AY23056)区

\section{Availability of data and materials}

The datasets used and/or analyzed during the current study are not publicly available but are available from the corresponding author on reasonable request.

\section{Ethics approval and consent to participate}

Because this was a prospective study, all subjects were required to sign an informed consent form. The study complied with the Declaration of Helsinki and was approved by the ethics committee of the second affiliated hospital of Jiaxing University (no. 20181102H02).

Consent for publication: Not applicable

\section{Competing interests}

We declare that we have no conflict of interest.

\section{Authors' details}

1 Department of Cardiology, The Second Affiliated Hospital of Jiaxing University, Jiaxing, Zhejiang Province, China

2 Medical College, Jiaxing University, Jiaxing, Zhejiang Province, China

\section{References}

1. Lee MS, Banka G. In-stent Restenosis. Interventional cardiology clinics. 2016;5(2):211-20.

2. Dangas GD, Claessen BE, Caixeta A, Sanidas EA, Mintz GS, Mehran R. In-Stent Restenosis in the Drug-Eluting Stent Era. J Am Coll Cardiol. 2010;56(23):1897-907.

3. Mehran RDG, Abizaid AS, Mintz GS, Lansky AJ, Satler LF, Pichard AD, Kent KM, Stone GW, Leon MB. Angiographic Patterns of In-Stent Restenosis Classification and Implications for Long-Term Outcome. Circulation. 1999;1999(100):1872-8.

4. Kimura T, Morimoto T, Nakagawa Y, Kawai K, Miyazaki S, Muramatsu T, Shiode N, Namura M, Sone T, Oshima S, et al. Very Late Stent Thrombosis and Late Target Lesion Revascularization After Sirolimus-Eluting Stent Implantation: Five-Year Outcome of the j-Cypher Registry. Circulation. 2011;125(4):584-91. 
5. Leon MB, Nikolsky E, Cutlip DE, Mauri L, Liberman H, Wilson H, Patterson J, Moses J, Kandzari DE. Improved Late Clinical Safety With Zotarolimus-Eluting Stents Compared With Paclitaxel-Eluting Stents in Patients With De Novo Coronary Lesions. JACC: Cardiovascular Interventions. 2010;3(10):1043-50.

6. Jukema JW, Verschuren JJ, Ahmed TA, Quax PH. Restenosis after PCI. Part 1: pathophysiology and risk factors. Nature reviews Cardiology. 2011;9(1):53-62.

7. Minacapelli A, Piraino D, Buccheri D, Cortese B. Drug-coated balloons for the treatment of in-stent restenosis in diabetic patients: A review of currently available scientific data. Catheterization cardiovascular interventions: official journal of the Society for Cardiac Angiography Interventions. 2018;92(1):E20-7.

8. Paraskevas KI, Baker DM, Pompella A, Mikhailidis DP. Does diabetes mellitus play a role in restenosis and patency rates following lower extremity peripheral arterial revascularization? A critical overview. Ann Vasc Surg. 2008;22(3):481-91.

9. Jimenez-Quevedo P, Suzuki N, Corros C, Ferrer C, Angiolillo DJ, Alfonso F, Hernandez-Antolin R, Banuelos C, Escaned J, Fernandez C, et al. Vessel shrinkage as a sign of atherosclerosis progression in type 2 diabetes: a serial intravascular ultrasound analysis. Diabetes. 2009;58(1):209-14.

10. Codner P, Gurm HS, Motivala A. Coronary Stents in Diabetic Patients: State of the Knowledge. Curr Cardiol Rep. 2017;19(4):28.

11. Sarembock IJ. Stent restenosis and the use of drug-eluting stents in patients with diabetes mellitus. Curr Diabetes Rep. 2004;4(1):139.

12. Qin Z, Zhou K, Li YP, Wang JL, Cheng WJ, Hu CP, Shi C, He H, Zhou YJ. Remnant lipoproteins play an important role of in-stent restenosis in type 2 diabetes undergoing percutaneous coronary intervention: a single-centre observational cohort study. Cardiovasc Diabetol. 2019;18(1):11.

13. Levine GN, Bates ER, Blankenship JC, Bailey SR, Bittl JA, Cercek B, Chambers CE, Ellis SG, Guyton RA, Hollenberg SM, et al. 2011 ACCF/AHA/SCAI Guideline for Percutaneous Coronary Intervention. A report of the American College of Cardiology Foundation/American Heart Association Task Force on Practice Guidelines and the Society for Cardiovascular Angiography and Interventions. J Am Coll Cardiol. 2011;58(24):e44-122.

14. Mak KH, Faxon DP. Clinical studies on coronary revascularization in patients with type 2 diabetes. Eur Heart J. 2003;24(12):1087-103.

15. Qin SY, Zhou Y, Jiang HX, Hu BL, Tao L, Xie MZ. The association of diabetes mellitus with clinical outcomes after coronary stenting: a meta-analysis. PloS one. 2013;8(9):e72710.

16. Konigstein M, Ben-Yehuda O, Smits PC, Love MP, Banai S, Perlman GY, Golomb M, Ozan MO, Liu M, Leon MB, et al. Outcomes Among Diabetic Patients Undergoing Percutaneous Coronary Intervention With Contemporary Drug-Eluting Stents: Analysis From the BIONICS Randomized Trial. JACC Cardiovascular interventions. 2018;11(24):2467-76.

17. Anfossi G, Trovati M. Pathophysiology of platelet resistance to anti-aggregating agents in insulin resistance and type 2 diabetes: implications for anti-aggregating therapy. Cardiovasc Hematol Agents Med Chem. 2006;4(2):111-28.

18. Tzoulaki I, Murray GD, Lee AJ, Rumley A, Lowe GD, Fowkes FG. Inflammatory, haemostatic, and rheological markers for incident peripheral arterial disease: Edinburgh Artery Study. Eur Heart J. 2007;28(3):354-62.

19. Prasad K. C-reactive protein (CRP)-lowering agents. Cardiovasc Drug Rev. 2006;24(1):33-50.

20. Song W, Ergul A. Type-2 diabetes-induced changes in vascular extracellular matrix gene expression: relation to vessel size. Cardiovasc Diabetol. 2006;5:3.

21. Nathan DM, Cleary PA, Backlund JY, Genuth SM, Lachin JM, Orchard TJ, Raskin P, Zinman B, Diabetes C, Complications Trial/Epidemiology of Diabetes I et al. Intensive diabetes treatment and cardiovascular disease in patients with type 1 diabetes. The New England journal of medicine 2005, 353(25):2643-2653.

22. Armstrong EJ, Waltenberger J, Rogers JH. Percutaneous coronary intervention in patients with diabetes: current concepts and future directions. J Diabetes Sci Technol. 2014;8(3):581-9.

23. Lemesle G, Bonello L, de Labriolle A, Maluenda G, Syed Al, Collins SD, Ben-Dor I, Torguson R, Kaneshige K, Xue Z, et al. Prognostic value of hemoglobin A1C levels in patients with diabetes mellitus undergoing percutaneous coronary intervention with stent implantation. The American journal of cardiology. 2009;104(1):41-5.

24. Wanner C, Krane V. Recent advances in the treatment of atherogenic dyslipidemia in type 2 diabetes mellitus. Kidney blood pressure research. 2011;34(4):209-17.

25. Qin Z, Zheng FW, Zeng C, Zhou K, Geng Y, Wang JL, Li YP, Ji QW, Zhou YJ. Elevated Levels of Very Low-density Lipoprotein Cholesterol Independently Associated with In-stent Restenosis in Diabetic Patients after Drug-eluting Stent Implantation. Chin Med J (Engl).

2017;130(19):2326-32.

Page 17/23 


\section{Figures}
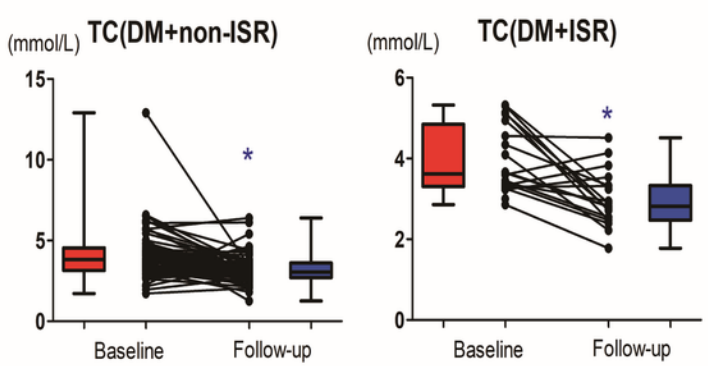

TC/HDL-c(DM+non-ISR)

TC/HDL-C(DM+ISR)
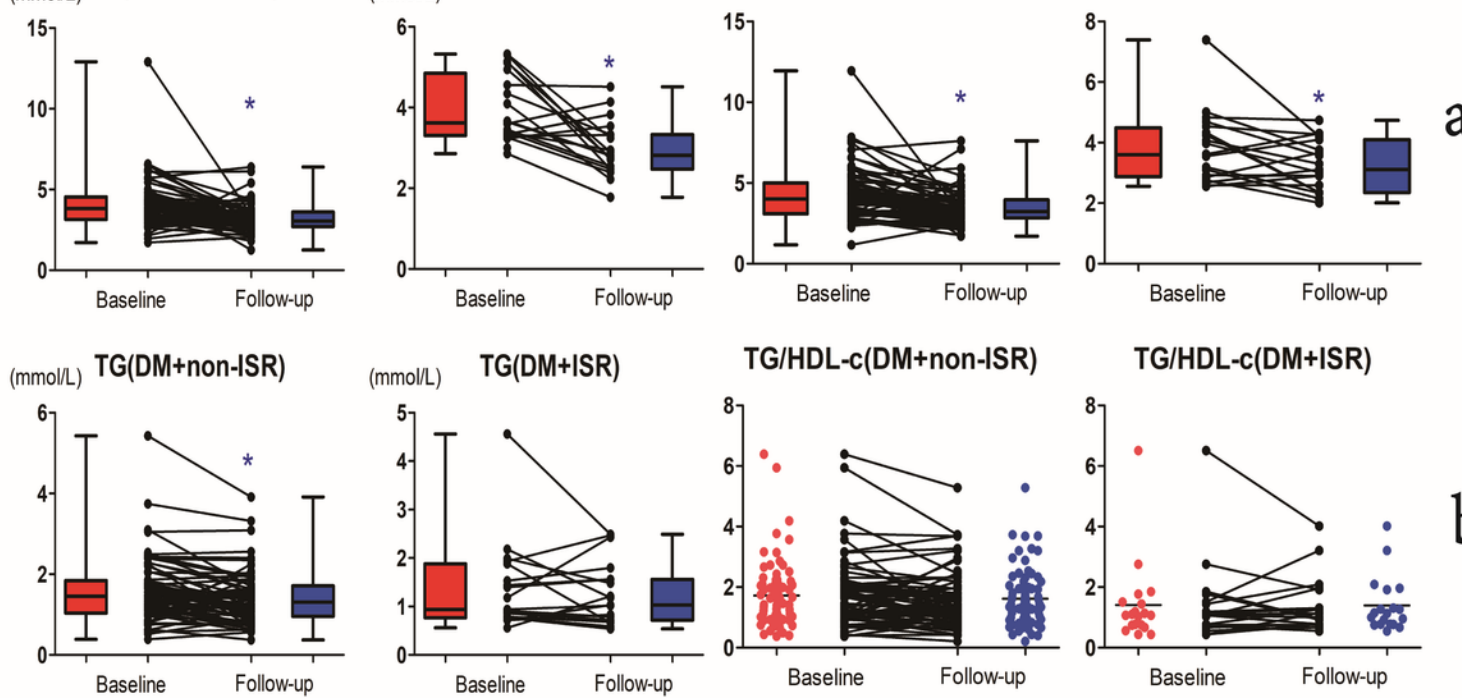

TG/HDL-c(DM+non-ISR)

TG/HDL-c(DM+ISR)
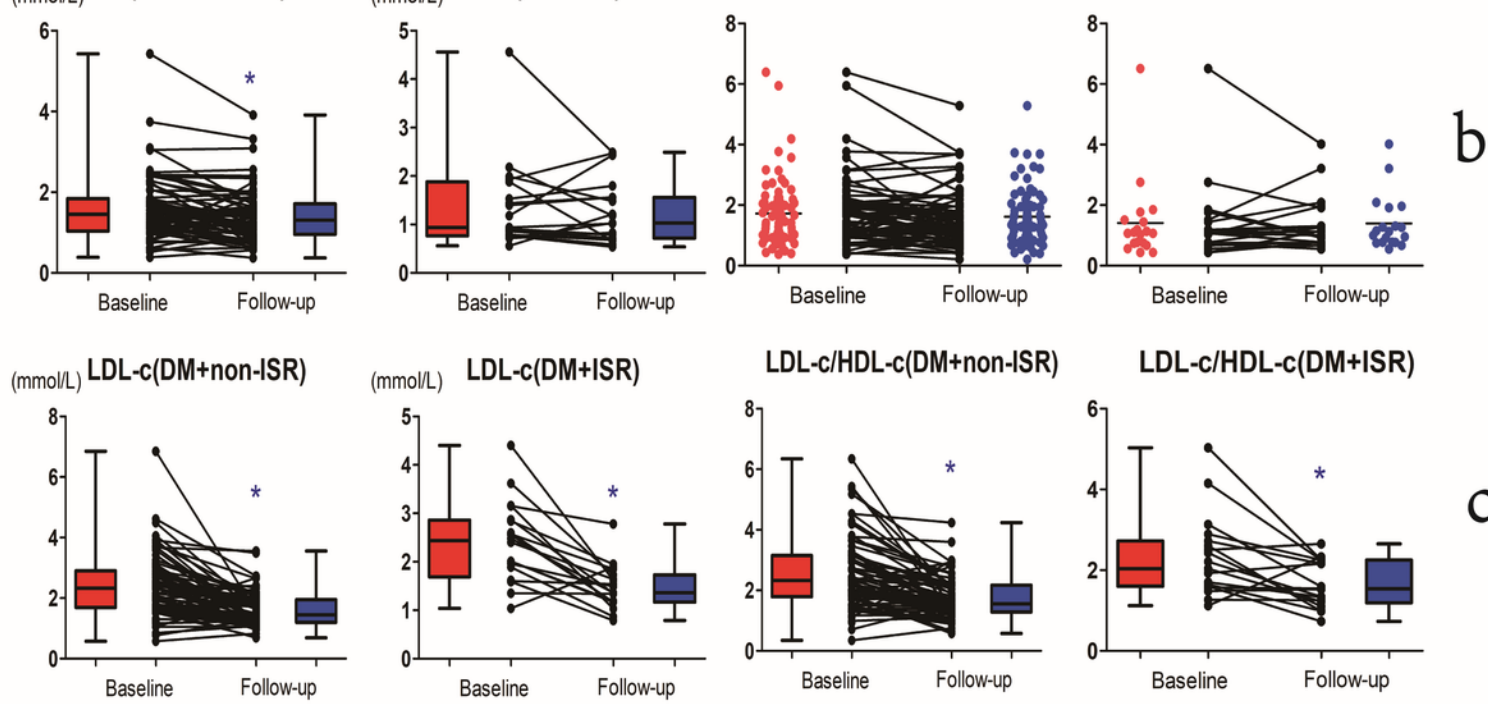

LDL-c/HDL-c(DM+non-ISR)

LDL-c/HDL-c(DM+ISR)
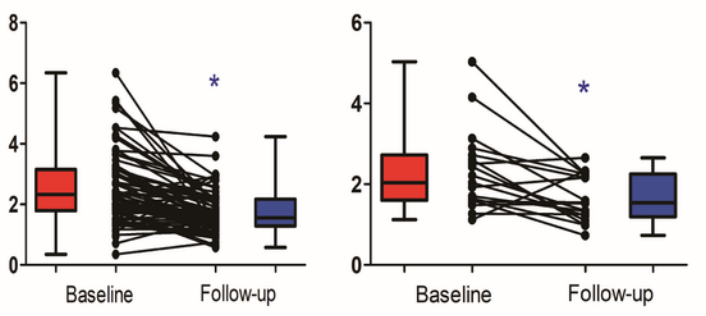

C
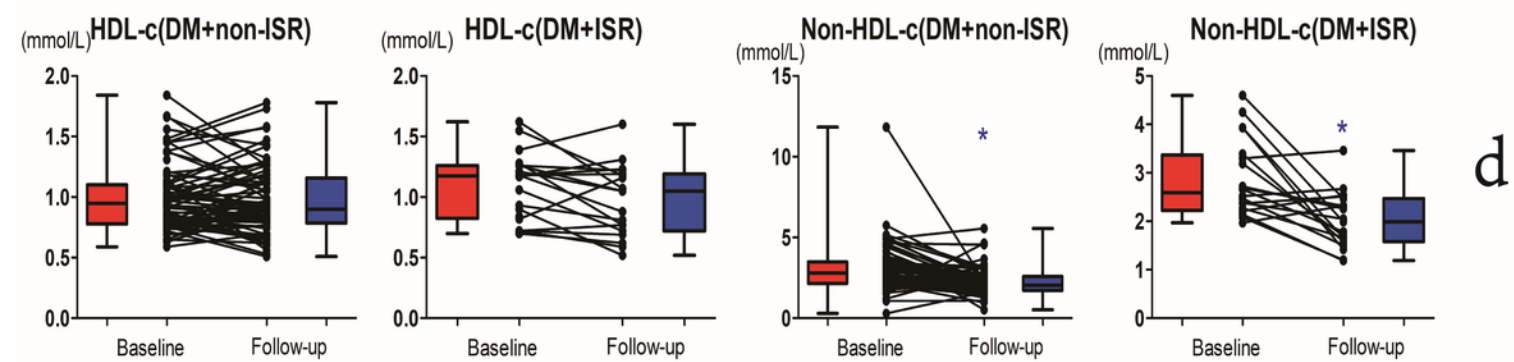

Figure 1

Comparison of blood lipid profile changes between two groups before PCl and before re-examination. Note: TC, total cholesterol; TG, triglycerides; HDL-c, high-density lipoprotein cholesterol; LDL-c, low-density lipoprotein cholesterol; ApoA1, Apolipoprotein A1; ApoB100, apolipoprotein B100; ApoE, apolipoprotein E; Non-HDL-c, non-high-density lipoprotein cholestasis. Baseline, pre-PCl; Follow-up, repeat CAG. * $P<0.05$. 

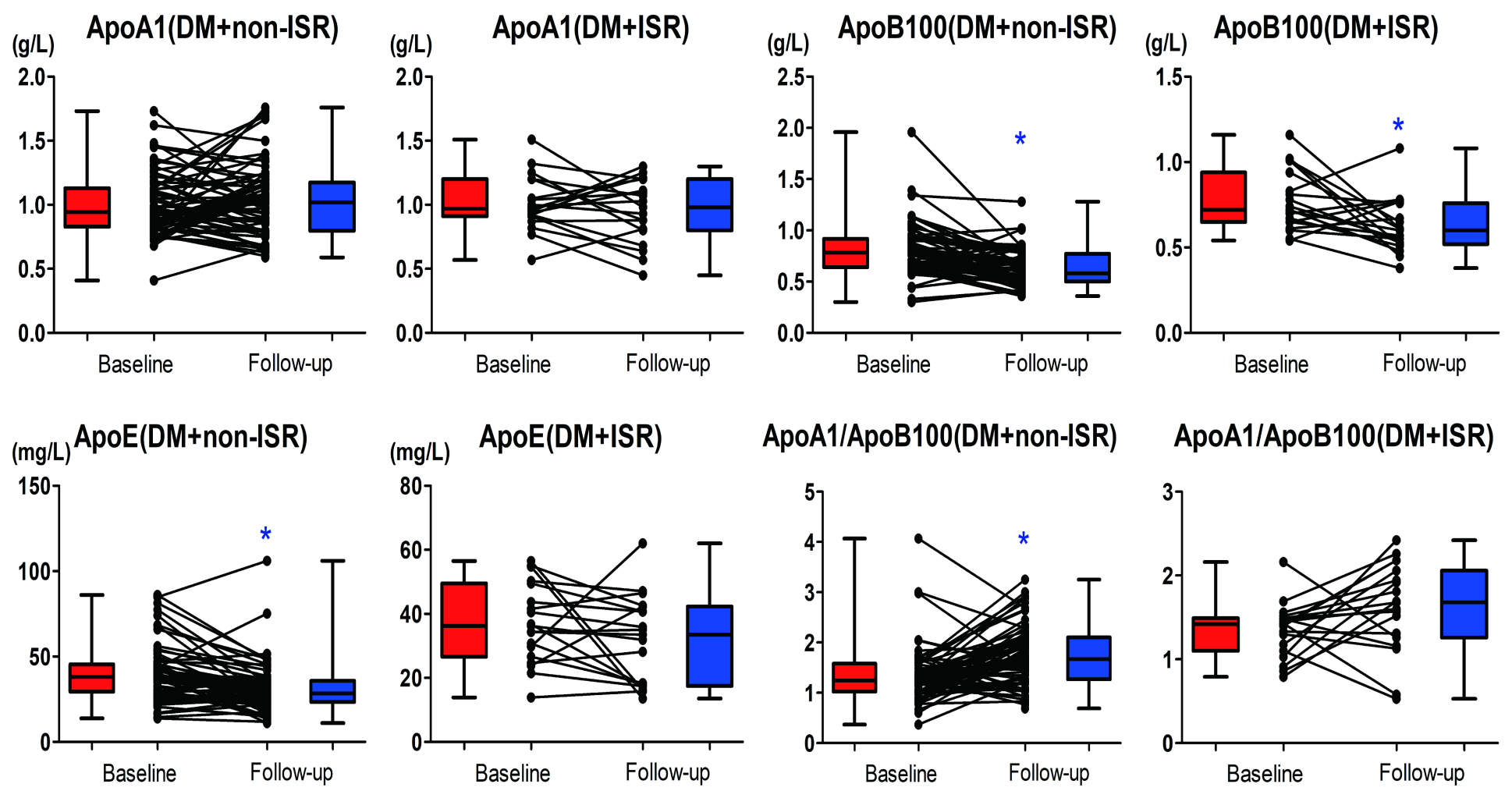

ApoA1/ApoB100(DM+non-ISR)

ApoA1/ApoB100(DM+ISR)
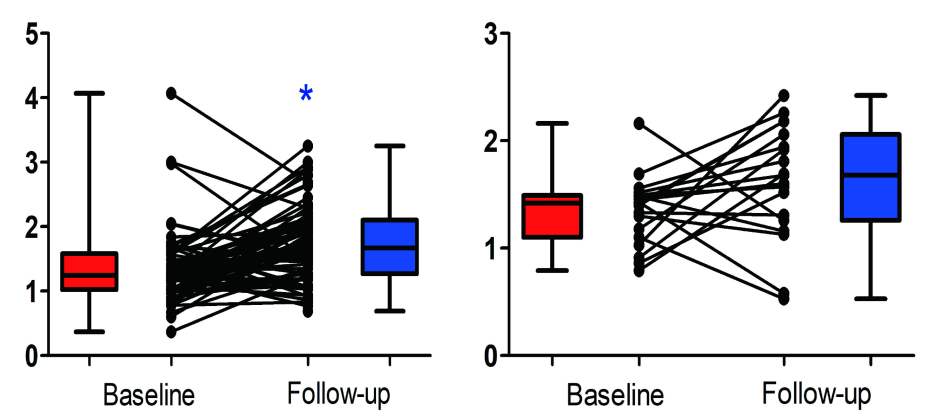

Figure 2

Comparison of blood apolipoprotein changes between the two groups before PCl and before re-examination. Note: ApoA1 , Apolipoprotein A1; ApoB100, apolipoprotein B100; ApoE, apolipoprotein E; * P <0.05. 

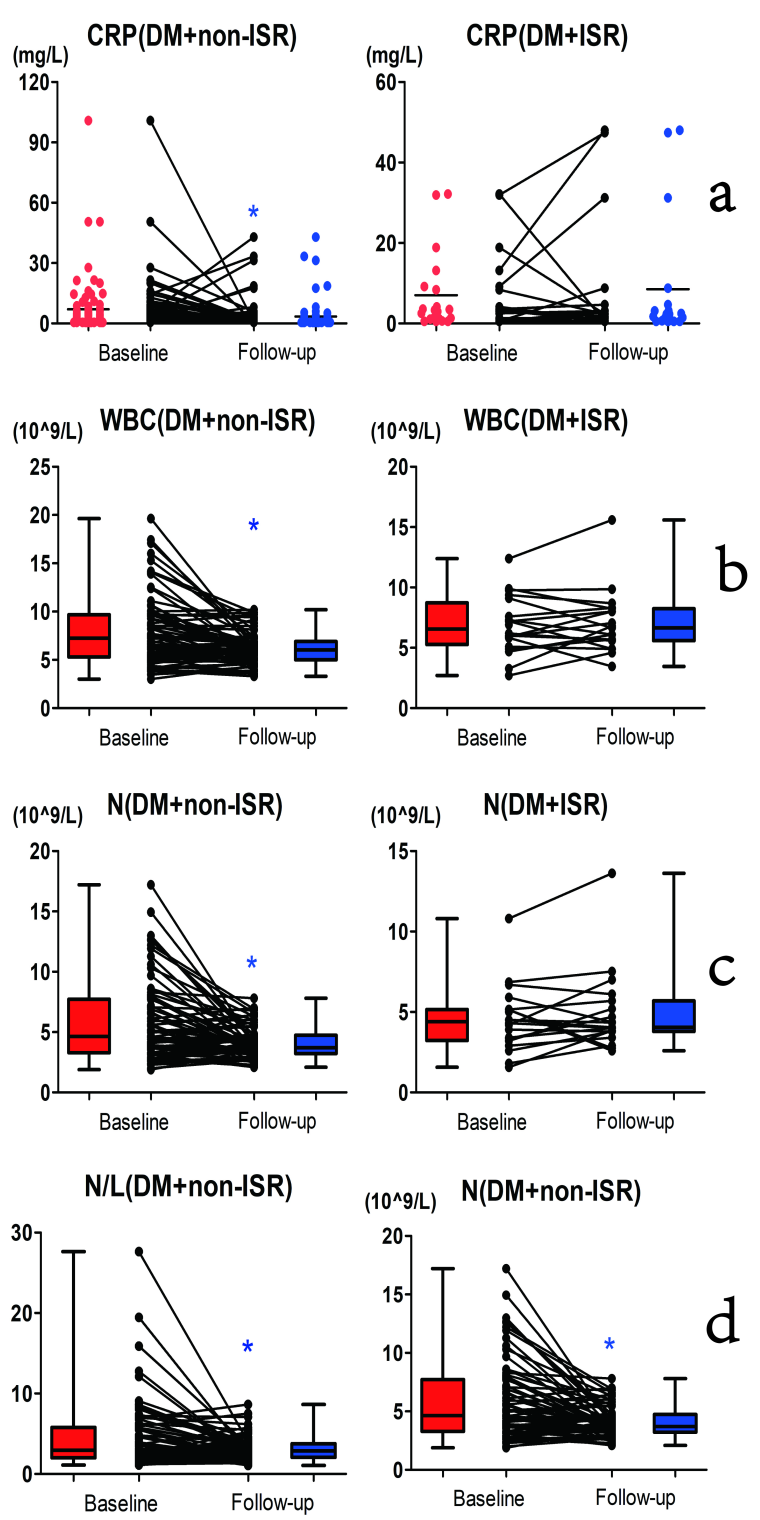

\section{Figure 3}

Comparison of inflammation indexes between the two groups before PCl and before re-examination. Note: CRP, C-reactive protein; WBC, white blood cells; N, neutrophils; L, lymphocytes. * $\mathrm{P}<0.05$. Baseline, pre-PCl; Follow-up, repeat CAG. 

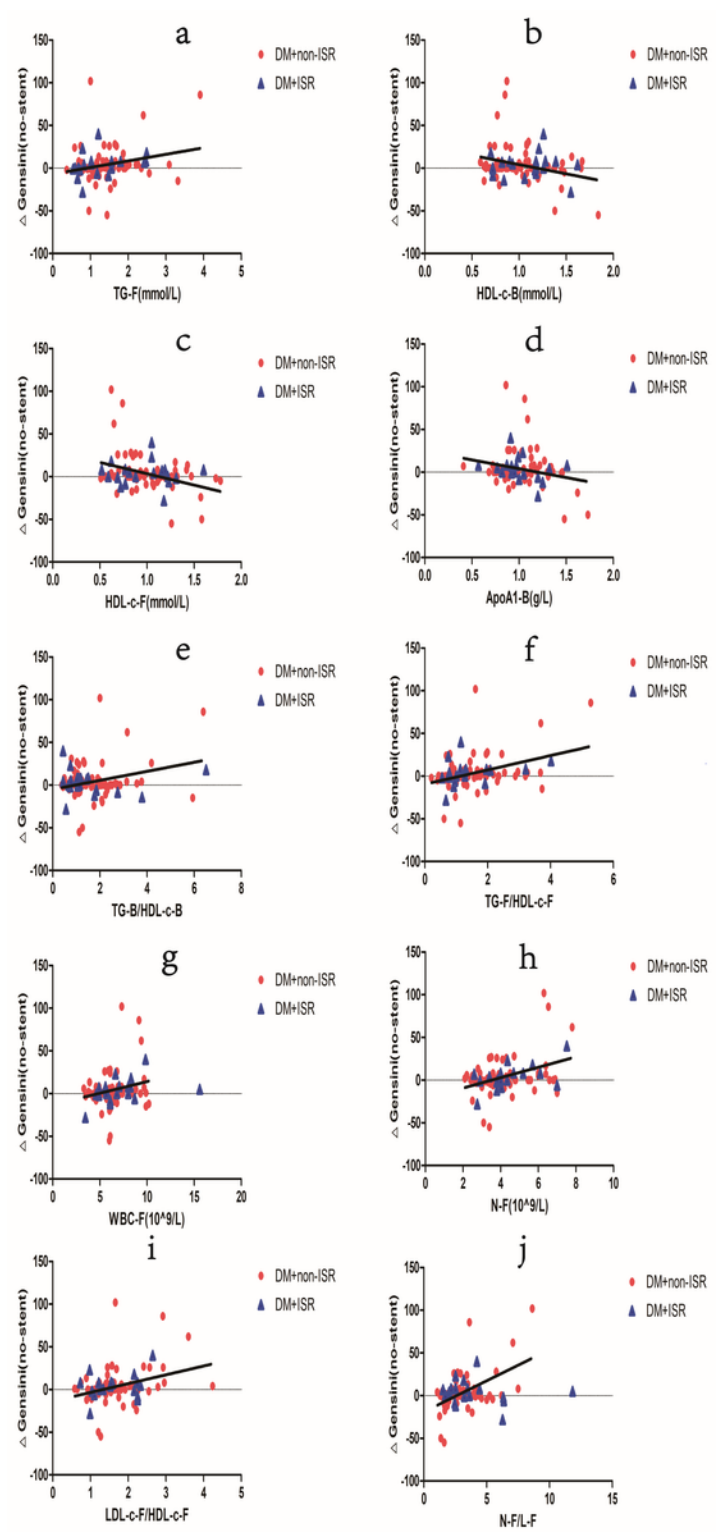

\section{Figure 4}

Linear correlation analysis of risk factors and $\triangle$ Gensini Note: TG, triglyceride; HDL-c, high-density lipoprotein cholesterol; ApoA1, apolipoprotein A1; LDL-C, low-density lipoprotein cholestasis; N, neutrophils; L, lymphocytes. B: Before PCl; F, before reviewing coronary angiography. 


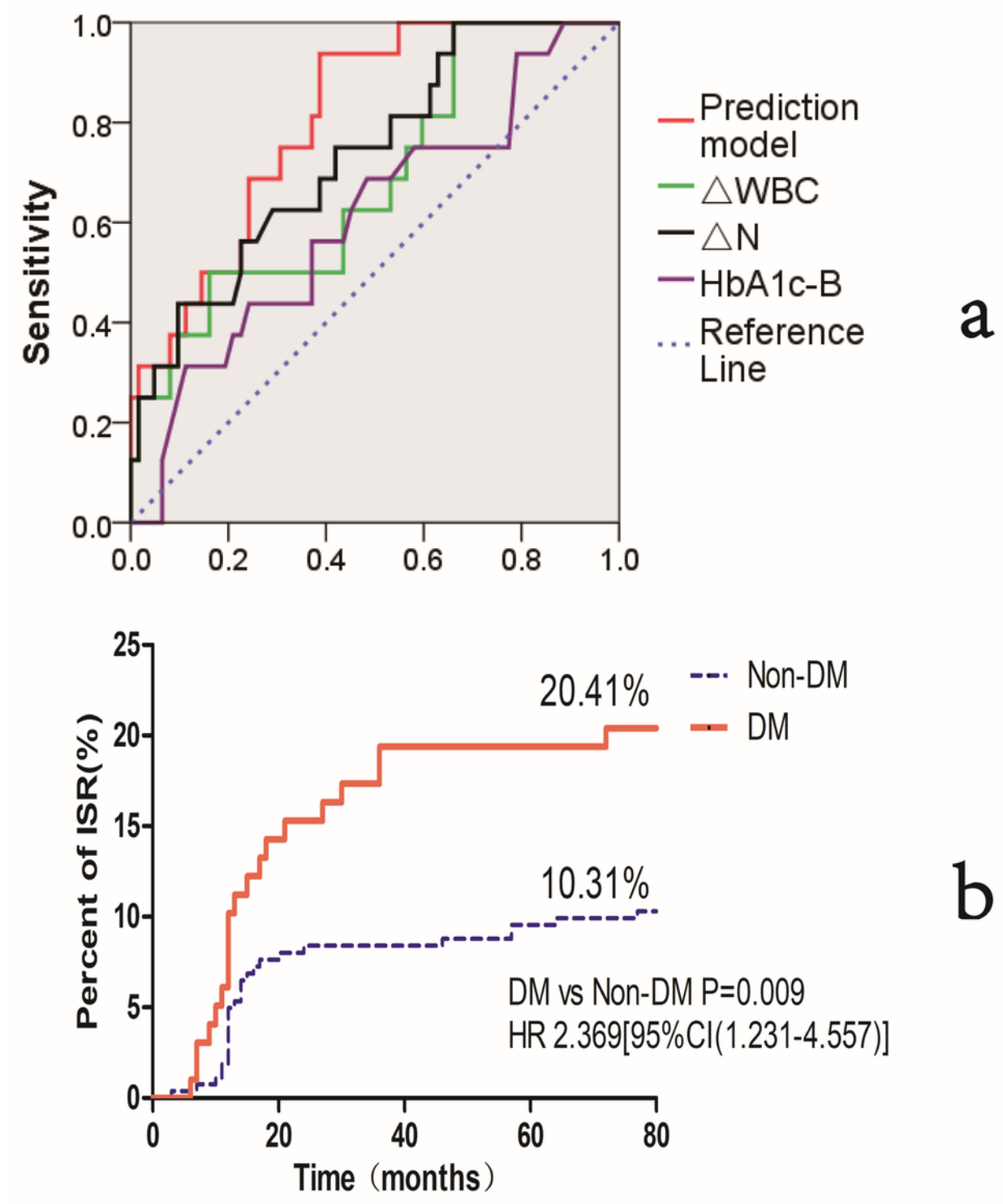

\section{Figure 5}

a. Predictive values of various factors for restenosis risk by ROC curve analysis. Predictive values of $\triangle N, \triangle W B C, H b A 1 c-B$ and restenosis risk prediction model for restenosis risk, which were evaluated by ROC. HbA1c, glycated haemoglobin A1c; WBC, white blood cells; N, neutrophils; $\mathrm{B}$, pre-PCI period; restenosis risk prediction model. $\mathrm{P}=[\exp (-4.842+0.562(\triangle \mathrm{N})+0.473(\mathrm{HbA} 1 \mathrm{c} 1-\mathrm{B}))] /[1+\exp (-4.842+0.562$ $(\triangle N)+0.473(\mathrm{HbA1c1-B}))]$; b. Kaplan-Meier plot of cumulative incidence of ISR in diabetic and non-diabetic groups. 


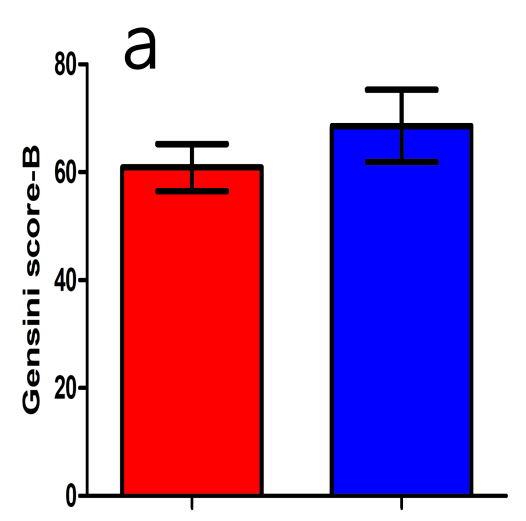

LDL-c Achieved LDL-c Failure

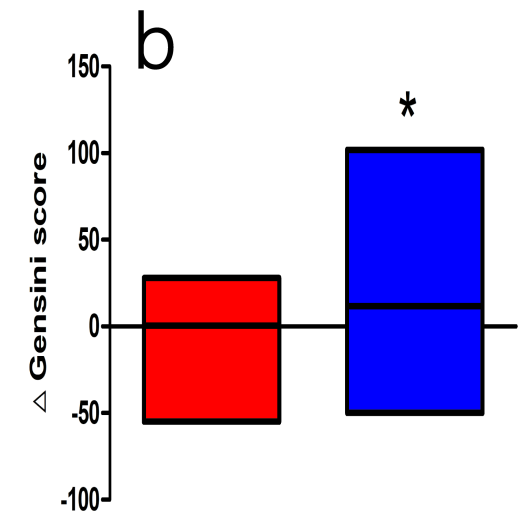

LDL-c Achieved LDL-c Failure

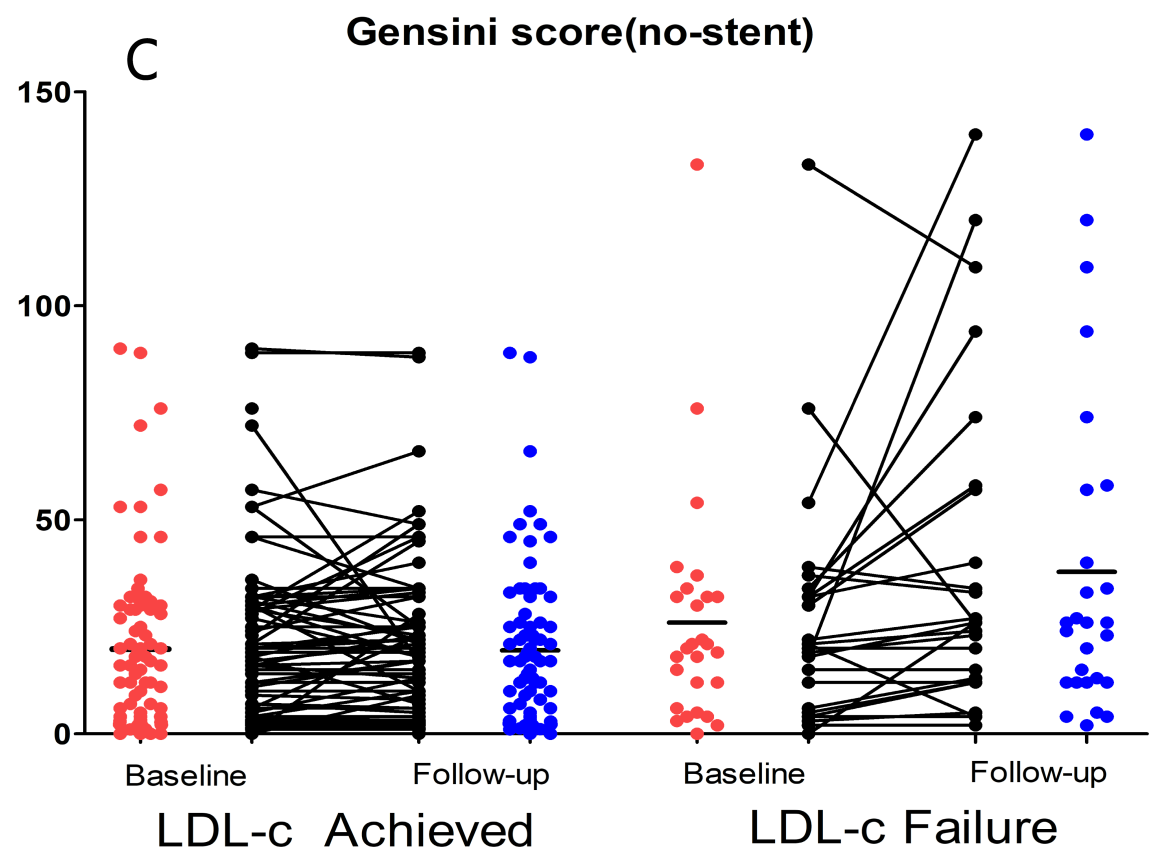

Figure 6

Relationship between LDL-c control status and Gensini progression. A: Comparison of the baseline of Gensini scores at pre-PCl period between LDL-c achieved group and LDL-c failure group; B: Comparison of difference in Gensini scores(no-stent) change between LDL-c achieved group and LDL-c failure group; C: LDL-c Trend graph of Gensini scores(no-stent) of both LDL-c achieved group and LDL-c failure group. Baseline: pre-PCl; Follow-up: Repeat coronary angiography. ${ }^{*}<0.05$.

\section{Supplementary Files}

This is a list of supplementary files associated with this preprint. Click to download.

- FIG1R01.PDF

- figure2.pdf 